\title{
Thermal Infrared Imaging Experiments of C-Type Asteroid 162173 Ryugu on Hayabusa2
}

\author{
Tatsuaki Okada $^{1,2}$ (D) Tetsuya Fukuhara ${ }^{3}$ Satoshi Tanaka ${ }^{1} \cdot$ Makoto Taguchi $^{3}$. \\ Takeshi Imamura ${ }^{4}$. Takehiko Arai ${ }^{1}$ Hiroki Senshu ${ }^{5}$. Yoshiko Ogawa ${ }^{6}$. \\ Hirohide Demura $^{6}$ - Kohei Kitazato ${ }^{6}$ - Ryosuke Nakamura ${ }^{7}$. Toru Kouyama ${ }^{7}$. \\ Tomohiko Sekiguchi $^{8}$ - Sunao Hasegawa ${ }^{1}$. Tsuneo Matsunaga ${ }^{9}$ Takehiko Wada ${ }^{1}$. \\ Jun Takita ${ }^{2}$ Naoya Sakatani ${ }^{1}$ - Yamato Horikawa ${ }^{10}$ • Ken Endo ${ }^{6}$ - Jörn Helbert ${ }^{11}$. \\ Thomas G. Müller ${ }^{12}$ • Axel Hagermann ${ }^{13}$
}

Received: 24 September 2015 / Accepted: 31 August 2016 / Published online: 27 September 2016

(C) The Author(s) 2016. This article is published with open access at Springerlink.com

\begin{abstract}
The thermal infrared imager TIR onboard Hayabusa2 has been developed to investigate thermo-physical properties of C-type, near-Earth asteroid 162173 Ryugu. TIR is one of the remote science instruments on Hayabusa2 designed to understand the nature of a volatile-rich solar system small body, but it also has significant mission objectives to provide information on surface physical properties and conditions for sampling site selection
\end{abstract}

$凶$ T. Okada

okada@planeta.sci.isas.jaxa.jp

1 Institute of Space and Astronautical Science, Japan Aerospace Exploration Agency, Sagamihara, Japan

2 Graduate School of Science, The University of Tokyo, Bunkyo Tokyo, Japan

3 Rikkyo University, Tokyo, Japan

4 Graduate School of Frontiers Sciences, The University of Tokyo Kashiwa, Japan

5 Planetary Exploration Research Center, Chiba Institute of Technology, Narashino, Japan

6 Center for Advanced Information Science and Technology, University of Aizu, Aizu-Wakamatsu, Japan

7 National Institute for Advanced Industrial Science and Technology, Tokyo, Japan

8 Hokkaido University of Education, Asahikawa, Japan

9 Center for Global Environmental Research, National Institute for Environmental Studies, Tsukuba, Japan

10 Department of Space and Astronautical Science, School of Physical Sciences, Graduate University for Advanced Studies, Sagamihara, Japan

11 Institute of Planetary Research, German Aerospace Center (DLR), Berlin, Germany

12 Max-Planck Institute for Extraterrestrial Physics, Garching, Germany

13 The Open University, Milton Keynes, UK 
as well as the assessment of safe landing operations. TIR is based on a two-dimensional uncooled micro-bolometer array inherited from the Longwave Infrared Camera LIR on Akatsuki (Fukuhara et al., 2011). TIR takes images of thermal infrared emission in 8 to $12 \mu \mathrm{m}$ with a field of view of $16 \times 12^{\circ}$ and a spatial resolution of $0.05^{\circ}$ per pixel. TIR covers the temperature range from 150 to $460 \mathrm{~K}$, including the well calibrated range from 230 to $420 \mathrm{~K}$. Temperature accuracy is within $2 \mathrm{~K}$ or better for summed images, and the relative accuracy or noise equivalent temperature difference (NETD) at each of pixels is $0.4 \mathrm{~K}$ or lower for the well-calibrated temperature range. TIR takes a couple of images with shutter open and closed, the corresponding dark frame, and provides a true thermal image by dark frame subtraction. Data processing involves summation of multiple images, image processing including the StarPixel compression (Hihara et al., 2014), and transfer to the data recorder in the spacecraft digital electronics (DE). We report the scientific and mission objectives of TIR, the requirements and constraints for the instrument specifications, the designed instrumentation and the pre-flight and in-flight performances of TIR, as well as its observation plan during the Hayabusa2 mission.

Keywords Thermography · Thermal inertia · Temperature · Near-Earth asteroid · Planetary exploration

\section{Introduction}

The thermal infrared imager TIR has been developed for Hayabusa2, a sample return mission to C-type near-Earth asteroid 162173 Ryugu (formerly $1999 \mathrm{JU}_{3}$ ), to image the thermal emission of the surface of the asteroid and investigate its surface thermo-physical properties. This thermographic instrument is based on a two-dimensional uncooled micro-bolometer array, inherited from the Longwave Infrared Camera LIR on the Akatsuki (Planet-C) Venus climate orbiter (Fukuhara et al. 2011). Thermal radiometry has been one of the traditional methods in planetary missions to measure temperature and surface physical properties of planetary surfaces, and thermal mapping has been conducted by tracking or scanning the surface from orbit (e.g., Kieffer et al. 1973; Chase et al. 1976; Kieffer et al. 1977; Christensen et al. 2001, 2003; Paige et al. 2009; Hiesinger and Helbert 2010). Recent progress in two-dimensional detectors in thermal infrared wavelengths enables us to take thermal images of the target asteroid at a glance. This is a new tool in planetary missions to measure the thermo-physical properties of the uppermost planetary surface, and we adopt this tool both for scientific and mission purposes on Hayabusa2.

C-type asteroids are primitive and the most abundant type of asteroids in the solar system that preserves a clue to understanding the origin and evolution of the solar system, since it is less evolved (less thermally altered) due to its smaller size compared to planets and large moons. A significant physical property that would be still preserved in most primitive asteroids is the physical condition, involving packing, porosity, and grain size distribution. In the planetary formation scenario, fine grains such as silicate dusts and ices in the proto-solar nebula were accreted to form small bodies called planetesimals, which should have been porous and loosely bound rubble-pile objects, several to tens of kilometers in diameter. Planetesimals were destructed into fragments and coalesced in impact events, and grew consequently to form larger bodies like planets. The interiors of the growing bodies were pressurized and compacted by meteor impact events and gravitational lithostatic force to become denser, less porous bodies. In the larger bodies, aqueous and thermal alteration processes should have occurred due to their volatile-rich composition and internal 
heat sources by radioactive nuclides. However, smaller primitive bodies might have avoided the occurrence of such pressurized or altered processes and still remain in a relatively primordial condition. Icy components could have been possibly lost by sublimation to form very porous and fluffy textures. Indeed, asteroid 953 Mathilde, the only C-type asteroid ever explored by NASA's Near-Earth Asteroid Rendezvous Shoemaker mission, has a low density of $1300 \pm 300 \mathrm{~kg} \mathrm{~m}^{-3}$ and on its surface, huge craters of hemispheric scale were found, which could be caused by meteor impacts into the loosely bound materials (Veverka et al. 1999).

The physical state of the surface is informed through thermal imaging, or thermography. Thermo-physical properties of the uppermost surface are characterized by grain size, porosity, or packing of the surface materials. But direct evidence of thermo-physical properties of primitive bodies is limited despite recent explorations. Thermal inertia values for Comet 9P/Tempel-1 and Comet 103P/Hartley-2, although still controversial, have been estimated to be 50 or 150 , using the data of 3 to $5 \mu \mathrm{m}$ near-infrared spectroscopy taken during flybys by NASA's Deep Impact and EPOXI missions (Groussin et al. 2013), respectively. These values are consistent with relatively fluffy, porous materials. During the historic landing of the Rosetta/ Philae lander, the surface of Comet 67P/ Churyumov-Gerasimenko was observed to be harder than predicted (Biele et al. 2015; Spohn et al. 2015).

A C-type asteroid is categorized by a flat, featureless reflectance spectrum in visible to near infrared wavelengths, and considered to be the origin of carbonaceous chondrite meteorites. But a carbonaceous chondrite meteorite found on the Earth should be the most consolidated part of the parent body and might not preserve its original physical condition because of break-up during the ejection from the parent body or during entry into the terrestrial atmosphere. In addition, the surface of C-type asteroids should have undergone some degree of processing such as (i) dehydration due to heating by the Sun; (ii) gardening by meteor impacts and ejecta sedimentation; (iii) space weathering through spattering by solar wind irradiation, dissociation by solar ultraviolet photons, or micro-cratering by micrometeor impacts; and (iv) fracture by thermal fatigue from cyclic temperature changes caused by asteroid rotation. Thus we know something of $\mathrm{C}$-chondrite meteorites but we know little about $\mathrm{C}$-type asteroids without conducting detailed in situ observations and detailed analysis of returned samples.

The physical state of the surface, i.e. whether it is covered with fine soils, pebbles, or dense rocks, indicates its history of surface processes undergone in the past. Asteroids are typically less dense $\left(<1500 \mathrm{~kg} \mathrm{~m}^{-3}\right.$ for C-type, $2000 \mathrm{~kg} \mathrm{~m}^{-3}$ for S-type) than terrestrial rocks $\left(2500\right.$ to $3300 \mathrm{~kg} \mathrm{~m}^{-3}$ ), suggesting that the asteroid is very porous (Consolmagno et al. 2008). This may be caused by interstitial spaces between rocks or the existence of micro-porosity such as fine pores or cracks inside of rocks. Intense meteor impacts cause destruction of the asteroid body, followed by re-accretion of impact fragments to form smaller rubble-pile asteroids, occasionally with large boulders found on their surface, such as 25143 Itokawa (Fujiwara et al. 2006). Impact cratering produces ejecta sedimentation consisting of pebbles and finer grains, although most fine grains tend to be lost by ejection due to the low escape velocity. Impact cratering also causes shaking of the whole asteroid (asteroid quake) to trigger granular flow, resulting in the geological dichotomy with the existence of flat and rough terrains on the same asteroid like Itokawa (Yano et al. 2006). Dense rocks were formed inside the larger parent body by lithostatic pressure and thermal metamorphism, but they could be exposed on the asteroid surface by later fragmentation of the parent body by intense impact events.

The physical state of the surface can be derived from thermo-physical properties such as thermal inertia. Thermal inertia is defined as $\left(k \rho C_{P}\right)^{0.5}$ in the SI unit of TI $=\mathrm{J} \mathrm{m}^{-2} \mathrm{~K}^{-1} \mathrm{~s}^{-0.5}$, 
Table 1 Relations of thermal inertia to surface physical state

\begin{tabular}{lll}
\hline $\begin{array}{l}\text { Thermal inertia: I } \\
{\left[\mathrm{J}^{-2} \mathrm{~s}^{-0.5} \mathrm{~K}^{-1}\right]}\end{array}$ & Surface physical state & Example \\
\hline$\sim 10$ & Very fluffy, high porosity $(\sim 80 \%)$ & Ceres, Martian soils \\
$\sim 50$ & Fine sand $(d<100 \mu \mathrm{m})$ & Lunar regolith \\
$100 \sim 200$ & Sandy regolith $(d \sim \mathrm{mm})$ & Eros soil \\
$200 \sim 400$ & Pebbles $(d \sim \mathrm{cm})$ & Itokawa flat terrain (Muses-C Regio) \\
$400 \sim 1000$ & Boulders, rock fragments $(d<\mathrm{m})$ & Itokawa rough terrain \\
$1000 \sim 2000$ & Rocks with high porosity & \\
$2000 \sim$ & Monolithic rocks & \\
\hline
\end{tabular}

where $k$ is thermal conductivity of the material, $\rho$ is its density, and $C_{P}$ is its specific heat at constant pressure. For a material of compact density $\rho_{0}$ and porosity $\varphi$, the bulk density becomes $\rho=\rho_{0}(1-\varphi)$. More fluffy or porous materials (low $\rho$ ) which tend to have a low value of $k$ due to a small cross section for heat flow typically have lower thermal inertia. For lower thermal inertia, the surface on a rotating asteroid shows a sharper and larger increase and decrease of temperature at the dawn and the dusk areas, respectively. On the other hand, denser materials (high $\rho$ ) typically have higher thermal conductivity and have higher thermal inertia. In this case, the thermal energy sinks into the interior in the day time and is released from the interior during night time, so that the surface temperature shows fainter and weaker temperature variation with a delayed time of peak temperature. Thus, thermal inertia is the physical property that informs the surface conditions which can be measured from orbit. This is applicable to understanding and characterizing the surface scientifically, and also to constraining the rock distribution to avoid hazardous landing areas or examining the thermal environment for safe operation for spacecraft. A typical relation of thermal inertia to the surface physical state is shown in Table 1 (e.g., Delbo et al. 2007; Okada 2016).

We briefly describe thermal experiments to be conducted in the Hayabusa 2 mission in Sect. 2, explain the objectives and requirements for the thermal infrared imager TIR in Sect. 3, show the instrumentation of TIR in Sect. 4, display some performance characteristics of TIR in Sect. 5, mention future observation plans during the cruise and asteroid rendezvous phases in Sect. 6, and summarize this paper in Sect. 7.

\section{Thermal Experiments in Hayabusa2}

\subsection{Outline of Thermal Experiments on Hayabusa2}

On Hayabusa2, thermal experiments investigating the surface of C-type asteroid Ryugu will be carried out by both remote sensing and in situ. TIR will conduct thermal emission imaging observations from the Home Position, about $20 \mathrm{~km}$ altitude sunwards from the asteroid surface almost every week during the 1.5 -year-long asteroid rendezvous phase. TIR will continually take images of thermal emission of the whole asteroid surface in the 8 to $12 \mu \mathrm{m}$ wavelength band every 512 seconds, during one asteroid rotation which is 7.63-hours long. In addition, when the spacecraft approaches the surface, TIR will also take thermal images from a lower altitude position, and spatially higher resolved images will consequently be taken. Using the TIR data, a global thermo-physical model of the asteroid will be constructed. The radiometer (MARA) (Grott et al. 2016) on the surface lander (MASCOT) (Ho 
et al. 2016) deployed from Hayabusa2 will continuously measure the thermal emission off the surface at one location through day and night for two asteroid days. MARA data is complementary to that of TIR which observes mainly from the day side, and contributes to improving the thermo-physical model.

\subsection{Summary of Hayabusa2 Mission}

Hayabusa2 is the second asteroid explorer organized by the Japan Aerospace Exploration Agency (Tsuda et al. 2013; Tachibana et al. 2014; Okada 2014). It was successfully launched on 3 December 2014, and will rendezvous with near-Earth asteroid Ryugu in 2018 and perform a series of remote sensing, surface robot experiments, and impact experiments there, as well as collect a sample from the surface of the asteroid, then return the sample to Earth in 2020. Hayabusa 2 has five remote-sensing experiments including an optical imager (ONC), laser ranging (LIDAR), near infrared spectrometer covering the $3 \mu \mathrm{m}$ absorption band (NIRS3), thermal infrared imager (TIR), and radio science. Hayabusa2 also carries three small rovers MINERVA-II and a small lander MASCOT (Mobile Asteroid Surface Scout) developed in collaboration with European countries. MASCOT is a 10-kg sized hopping lander which has a wide angle imager (CAM), a 6-band thermal radiator (MARA), a 3-axis magnetometer (MAG), and a hyperspectral infrared microscope (MicrOmega). Hayabusa2 has a sampling device (SMP), and impact experiment devices including a small carry-on impactor (SCI) and a deployable camera (DCAM).

The science goals of Hayabusa2 aim to understand the origin and evolution of materials in the solar nebula and in the asteroid parent bodies, as well as to constrain the physical properties of small bodies during the planetary accretion processes. It is essential in Hayabusa2 to conduct global to local to micro-scale observations by exploiting the synergy of remote sensing, surface measurements, and analysis of returned samples.

\subsection{Target Asteroid Ryugu}

The target body to be visited by Hayabusa2 is 162173 Ryugu, and its characteristics are tabulated in Table 2 (e.g., Müller et al. 2011; Ishiguro et al. 2014). Ryugu is a C-type $\left(\mathrm{C}_{\mathrm{g}}\right)$ near-Earth asteroid, and its perihelion and aphelion is 0.96 and $1.42 \mathrm{AU}$, respectively. The body has a roughly rounded shape with about $0.9 \mathrm{~km}$ in diameter, as derived from visible and infrared ground observations. The asteroid's period of rotation is 7.63 hours. Its reflectance spectrum is relatively featureless and flat, indicating a C-type asteroid in taxonomy. Although most previous observations have a low signal-to-noise ratio, one measurement appears to show a faint absorption at the $0.7 \mu \mathrm{m}$ band (Vilas 2008), suggesting a $\mathrm{CM}$ chondrite-like material. Its averaged albedo is about 0.05 , consistent with the typical C-type asteroid. Its current best estimate of global thermal inertia is $250 \pm 50$ (TI), indicating the surface is unlikely to be covered with lunar-like fine regolith or monolithic dense large boulders, but more consistent with pebbles. The only C-type asteroid explored to date is the relatively large and low-density asteroid 953 Mathilde, considered to be a very loosely bound rubble-pile object. To date, we do not know whether Ryugu resembles Mathilde or is completely different from this known asteroid.

\subsection{Temperature Profile on Asteroid Surface}

The surface physical properties of the asteroid would indicate the history of surface processes undergone in the past, depending on whether the surface is covered with fine soils, 
Table 2 Characteristics of 162173 Ryugu (e.g., Müller et al. 2011; Ishiguro et al. 2014)

\begin{tabular}{ll}
\hline Taxonomic type & $\mathrm{C}_{\mathrm{g}}$ \\
Diameter & $0.87 \pm 0.03 \mathrm{~km}$ \\
Axis ratio & $a / b=1.0153, b / c=1.0135$ \\
Rotation period & $7.63 \pm 0.01$ hour \\
Pole axis $(\lambda, \beta)$ & $(329,-39) \pm 20^{\circ}$ \\
Geometric albedo $(\mathrm{Pv})$ & 0.05 \\
$\mathrm{H}$ & $18.82 \pm 0.021$ \\
$\mathrm{G}$ & $-0.110 \pm 0.007$ \\
Thermal inertia & $250 \pm 50 \mathrm{~J} \mathrm{~m}^{-2} \mathrm{~s}^{-0.5} \mathrm{~K}^{-1}$ \\
Orbit (solar distance) & $0.9633-1.4159 \mathrm{AU}$ \\
\hline
\end{tabular}

Fig. 1 Calculated temperature profiles as a function of surface thermal inertia for the equatorial region of an asteroid with a rotational period of 7.63 hours, where albedo $=0.05$, emissivity $=0.93$. Details are given in the text

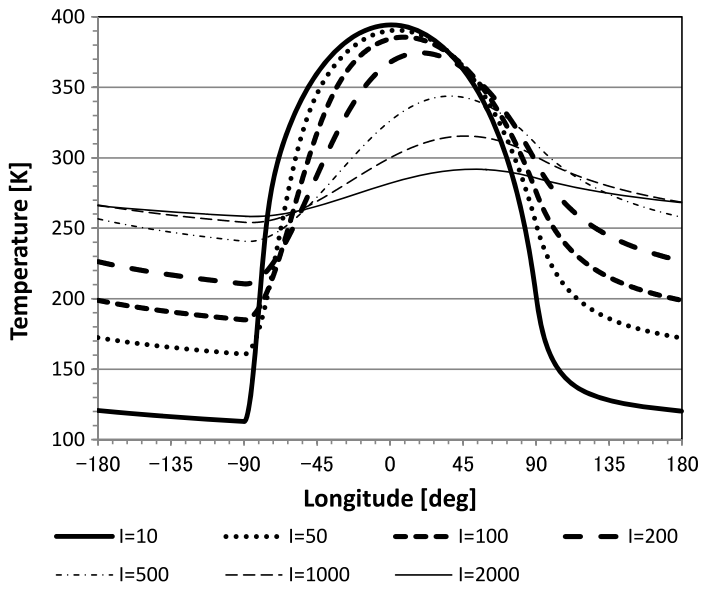

pebbles, porous boulders or dense rocks. Asteroids are typically less dense than terrestrial rocks, implying higher porosity.

Thermo-physical properties are highly affected by the physical state of the surface. Since the heat transfer through a porous or fluffy surface is small, the heat radiation from the Sun is deposited in the thin layer with low heat capacity at the uppermost surface and never penetrates to greater depths. In this case, the surface temperature increases sharply at dawn, but also decreases fast at dusk. On the other hand, heat transfer is larger in a denser, more conductive surface, and part of the heat input from the Sun sinks into the interior during daytime, but is released from there during nighttime. In this case, the temperature changes become rather moderate and the timing of peak temperature is delayed from the local noon.

Figure 1 shows an example of the temperature profile as a function of surface thermal inertia at the equatorial region of an asteroid with a rotational period of 7.63 hours, where the solar distance is $1 \mathrm{AU}$, the rotation pole is perpendicular to the asteroid orbital plane, and the surface albedo and emissivity are 0.05 and 0.93 , respectively. For the very fluffy surface case (the thermal inertia $I=10 \mathrm{TI}$ ), the peak temperature is about $400 \mathrm{~K}$ almost at noon, while the temperature becomes $110 \mathrm{~K}$ in the nighttime. For the case of pebbles ( $I=200 \mathrm{TI})$, the peak temperature is about $370 \mathrm{~K}$ in the afternoon at $18^{\circ}$ longitude while the temperature is higher than $210 \mathrm{~K}$ even during nighttime. For the dense rock case $(I=2000 \mathrm{TI})$, the temperature ranges between 250 and $300 \mathrm{~K}$ and the peak temperature is approximately at $45^{\circ}$ longitude. 
Table 3 Targets and the possible objectives for TIR observations

\begin{tabular}{|c|c|c|}
\hline Target & Description & Operation \\
\hline Boulders & Evolution of materials originated from parent body interior & LA, CU, HP \\
\hline $\begin{array}{l}\text { Crater walls and } \\
\text { interior }\end{array}$ & Direct probing of interior materials and structures & LA, HP \\
\hline Regolith & Flow and sedimentary processes under micro gravity & HP, LA, CU \\
\hline $\begin{array}{l}\text { Overall } \\
\text { thermo-physical } \\
\text { properties }\end{array}$ & Comparison with ground observation & HP, LA \\
\hline $\begin{array}{l}\text { Yarkovsky/Yorp } \\
\text { effects }\end{array}$ & Total mass, moment of inertia in asteroid & HP \\
\hline Phase function & $\begin{array}{l}\text { Updating TIR emission phase functions for roughness } \\
\text { modeling }\end{array}$ & HP \\
\hline \multicolumn{3}{|l|}{ (angular relation) } \\
\hline Moons & Orbiting satellite for gravity measurement & HP \\
\hline Dust clouds & Evidence of levitation, and existence of floating dust & HP \\
\hline Asteroid shape model & Asteroid shape modeling including night time & HP \\
\hline Geologic features & $\begin{array}{l}\text { Detection of features such as ejecta, sediments, ponds, buried } \\
\text { rocks }\end{array}$ & LA, HP \\
\hline SCI crater search & Finding the SCI impact crater using temperature difference & LA, CU, TD \\
\hline Touchdown (TD) site & Touchdown site selection and geologic context description & CU, TD \\
\hline
\end{tabular}

Operation range: HP: Home position $(10 \sim 20 \mathrm{~km})$, LA: Low altitude $(1 \sim 5 \mathrm{~km})$, CU: Close-up $(0.1 \sim 1 \mathrm{~km})$, TD: Touch-down $(<0.1 \mathrm{~km})$

We have conducted numerical studies with a thermal model of asteroid Ryugu as well as general cases for a variety of thermal inertias and pole inclinations in more detail, which will be reported in the companion paper (Takita et al. 2016).

\section{Objectives and Requirements of TIR}

\subsection{Scientific Objectives of TIR}

The main goals of TIR for the investigation of thermo-physical properties of the asteroid surface are (1) to understand the origin and evolution of asteroids and (2) to characterize the current state of a micro-gravitational small body. The targets and their possible objectives for TIR observations from various altitudes are shown in Table 3. In Fig. 2 we summarize the traceability map for the science objectives, observation targets, information requirements, and the performances required for TIR.

\subsubsection{Understanding the Origin and Evolution of Asteroids}

TIR will observe the asteroid structures found on the surface or inside the huge craters which are indicative of the asteroid formation processes. A homogeneously porous structure is likely in the event that the asteroid was formed by simple accretion growth of silicate dust and ice compounds, followed by sublimation of volatile components. On the other hand, a homogeneously dense structure is considered if the asteroid (or its parent body) has 


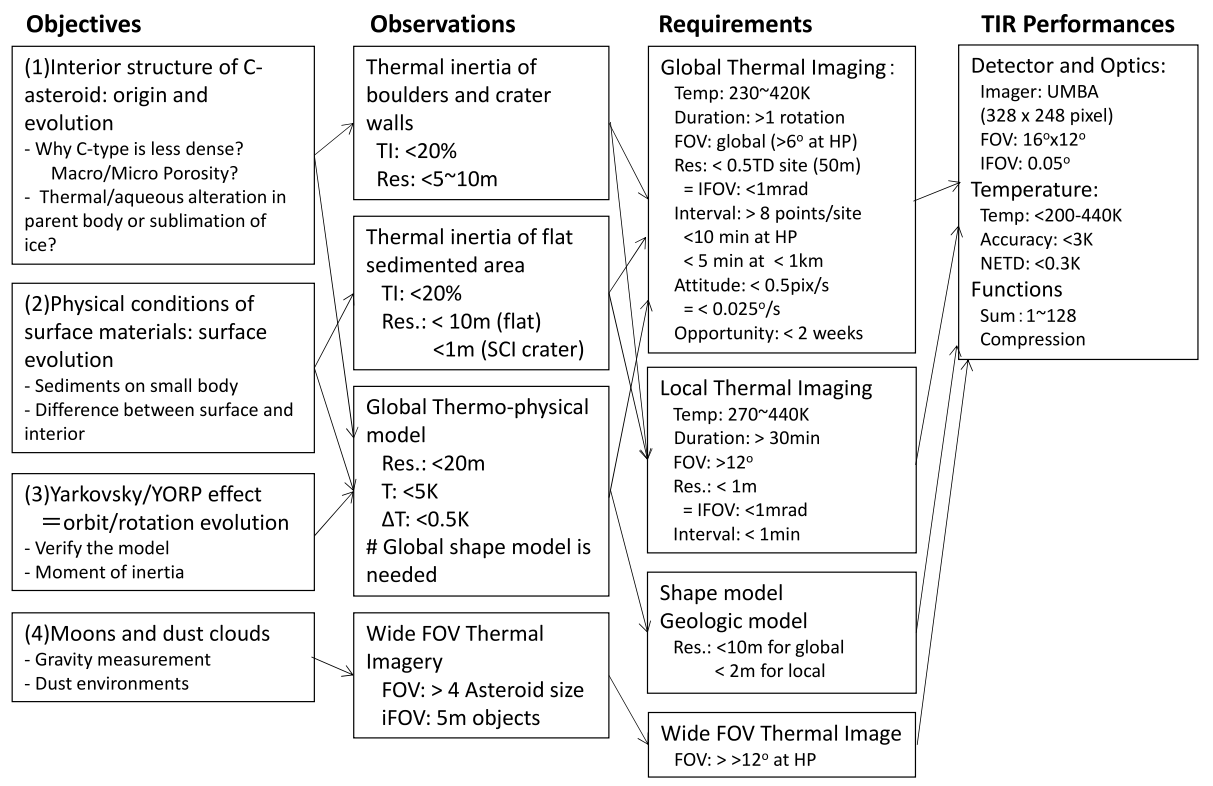

Fig. 2 Traceability map for science objectives, observations, information requirements, and TIR performance requirements

undergone a severe compaction process by impacts and thermal alteration after the body was accreted. Very heterogeneous features are expected if the asteroid is a rubble-pile object and composed of multiple boulders of various sizes that originated from impact fragments of an evolved parent body.

A thermal inertia map that shows the regional distribution of surface physical properties such as porosity or grain size lets us constrain the asteroid history. The thermal inertia of huge boulders implies a degree of past compression in the parent body. The thermal inertia of smooth flat area can deliver important information on the formation by ejecta sedimentation or granular flow under micro-gravity. The composition of surface materials cannot be determined solely by TIR but could be much improved if the thermal emission component estimated from TIR data is used for subtraction from the NIRS3 spectrum (Iwata et al. 2016).

\subsubsection{Characterizing the Current State of Micro-Gravitational Small Body}

The orbital and rotational evolution of solar system objects is affected by two thermal effects, the Yarkovsky effect and the YORP (Yarkovsky-O'Keefe-Radzievskii-Paddack) effect (e.g., Bottke et al. 2006). If an accurate thermo-physical model is constructed, the asteroid's density or moment of inertia is constrained. The relation of asteroid size to thermal inertia or typical thermal conductivity can be compiled from ground-based observations (Delbo et al. 2007). TIR will provide a disc-resolved thermal inertia map of Ryugu, which will be a good ground truth to verify and improve the thermal models of the asteroid. Thermal imagery can be a more powerful tool for detecting geologic features than optical imagery can, which is especially true for surfaces with varying porosity, such as crater ejecta, veins, grooves, or buried structures. Thermal emission from the dust surrounding the asteroid which might be 
supplied by electrostatic forces (levitation) or micro-meteor impacts might be detected by thermal imagery.

\subsection{Mission Objectives of TIR}

TIR data is also used for mission objectives such as landing site selection as well as safe descent operation for sample collection. In Fig. 3, we present the traceability map for mission objectives, observation targets, requirements for information, and the performance required for TIR, focused on conducting the descent and touchdown operations.

\subsubsection{Landing Site Selection}

Thermal inertia derived from TIR observations reflects the typical particle size of the regolith layer so that the landing site can be selected that is most suitable for sample collection from the viewpoint of particle size (1 mm diameter), even from Hayabusa2's Home Position. If particles are $1 \mathrm{~mm}$ in size, the sample is expected to contain most of the significant minerals and textures so that it is sufficiently informative for analysis. If particle size is less than $0.1 \mathrm{~mm}$, as was typical in the case of samples returned by Hayabusa, the sample may only include a limited subset of minerals and textures (Tachibana et al. 2014). On the other hand, the sampling device should collect samples much smaller than $10 \mathrm{~mm}$, so that the best size range for sample collection is about $1 \mathrm{~mm}$ in diameter. The thermal inertia map produced by TIR will help finding the best area with regard to grain size.

Using the thermal inertia map by TIR, the highest temperature ever experienced in the asteroid's history is estimated at a given depth for each site on the asteroid, according to a dynamically simulated trajectory of the entire lifetime of a near-Earth asteroid (Michel and Delbo 2010). This may be important for determining what kinds of organic matter are lost due to thermal processing or still remain on the surface of the asteroid, and may be one of the key pieces of information for landing site selection.

\subsubsection{Safe Operation for Descent of Spacecraft to the Surface}

The thermal emission energy of the surface of the asteroid as well as the surface temperature are measured by TIR, and also estimated at any given time using the thermo-physical model constructed by TIR observations. Thermal inertia maps are often used to estimate rock or boulder populations (Golombek et al. 2003). Prior to the descent and touchdown operation, it is important to assess whether the surface thermal environment and boulder distribution is hazardous for landing or not.

\subsection{Requirements for TIR Observation and Specification}

The desired performance of TIR is discussed in 2 and 3 for science and mission objectives, respectively. The spatial resolution expected during typical operation phases is shown in Table 4 , where IFOV $=1 \mathrm{mrad}$. Spatial resolution is subject to the following three requirements: (1) TD candidate site (flat area of $>50 \mathrm{~m}$ ) must be resolved by at least 2 pixels from $\mathrm{HP}(\mathrm{H}=20 \mathrm{~km})$, which is $<1.2 \mathrm{mrad}$ or $0.07^{\circ}$; (2) SCI crater (D $\left.>2 \mathrm{~m}\right)$ must be resolved by at least 2 pixels from the low altitude $(\mathrm{H}=1 \mathrm{~km})$, which is $<1 \mathrm{mrad}$ or $0.057^{\circ}$. The touchdown position touched by sampler horn $(\mathrm{D}=20 \mathrm{~cm})$ must be resolved by at least two pixels from $50 \mathrm{~m}$ altitude, which is $<2 \mathrm{mrad}$ or $0.11^{\circ}$. In summary, IFOV of TIR should be $<1 \mathrm{mrad}$. We summarize the requirements for TIR observation and specification in Table 5 . 
Table 4 Estimated spatial resolution for main operation phases of TIR assuming an IFOV of approx. $0.88 \mathrm{mrad}$

Observation phase Altitude [km] Spatial res. [m] Remarks

AP: Before arrival $\quad 2000 \sim 20 \quad 1700 \sim 17$

HP: Global features $\quad 20 \sim 10$

Mid-altitude:

Global and high res.

$\sim 5$

$\sim 4$

Low-altitude:

$$
\sim 1
$$

Local site features

TD: Local site, $<0.05$ high res.

XO: Larger phase angle
$20 \sim 10 \quad 17 \sim 9$
- Asteroid light-curve measurements, compared with ground observations

- Search for moons, observation of dust environments

- Image every several minutes for one rotation, once a week

- Solar phase angle $0-45^{\circ}$, solar distance 0.96-1.42 AU

- Several times, global coverage and higher resolution

- Every several minutes in one rotation

- $1 \mathrm{~h}$ observation before TD, Landers/SCI deployment

- $280 \mathrm{~m} \times 210 \mathrm{~m}$ area, SCI crater finding

- Several time for TD and TD rehearsals

- Highest resolution for each sites

- Large phase angle observation $\left(\theta \mathrm{s}>45^{\circ}\right)$

- From East-West, North-South

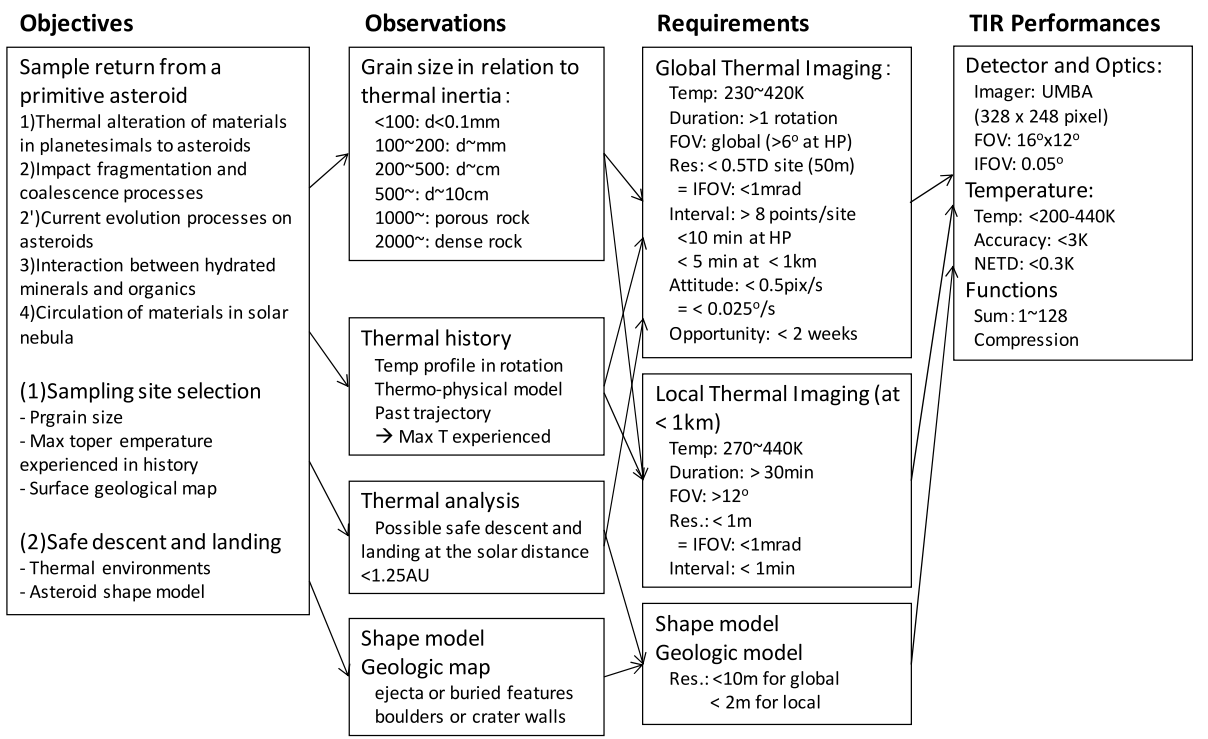

Fig. 3 Traceability map for mission objectives, observations, information requirements and TIR performance requirements, focused on conducting the descent and touchdown operations 
Table 5 Requirements for TIR observations and specifications for each objective

Objectives
Thermo-physical properties
of Boulder (originates from
parent body interior)
- density of C-type asteroid
is low
- thermal alteration in parent
body
- similarity to C-chondrites

Thermal inertia distribution of Crater interior (current interior structure)

- Interior density structure of 1999JU3

- Interior heterogeneity of 1999JU3

Thermal inertia map on regolith surface

- grain size distribution and formation of surface sedimentation

- regolith sticking or cohesion mechanism (for very fluffy case)

- thermal conduction under microgravity

\section{Comparison}

with ground-based asteroid

thermal model

- heterogeneity from surface

to interior

(formation process of

1999JU3)

- consistency with ground based TPM

- Verification of diameter-TI

relation by Delbo et al. (2007)

\section{Yarkovsky/YORP effects}

- Relation and comparison with thermal emission un-isotropy to evolution of orbit and rotation

- Asteroid mass,moment of inertia

Uppermost surface fine grains

- Existence and origin

of fine grain layer
Requirements for TIR

observation

Thermography of boulders

- $\mathrm{T}$ profile by rotation

(accuracy <5 K)

- different solar distance

- spatial resolution $<10 \mathrm{~m}$

- $\mathrm{T}$ profile by rotation

(accuracy <5 K)

- different solar distance

- spatial resolution $<10 \mathrm{~m}$

Global thermal mapping:

- T profile by rotation

(accuracy <5 K)

- different solar distance

- spatial resolution $<20 \mathrm{~m}$

Global shape model ( $<10 \mathrm{~m}$ res.)

Surface conditions informed by

ONC, MINERVA, MASCOT, sample

Requirements for TIR specification

FOV: $>6^{\circ} @ \mathrm{HP},>10^{\circ} @ 1 \mathrm{~km}$

IFOV: $<1 \mathrm{mrad} @ \mathrm{HP}, 1 \mathrm{~km}$

NETD: $<0.5 \mathrm{~K} @ 350 \mathrm{~K}$

$\Delta \mathrm{T}:<5 \mathrm{~K} @ 350 \mathrm{~K}$

FOV: $>3^{\circ} @ \mathrm{HP},>10^{\circ} @ 1 \mathrm{~km}$

IFOV: $<1 \mathrm{mrad} @ \mathrm{HP}$

NETD: <0.5 K @350 K

$\Delta \mathrm{T}:<5 \mathrm{~K} @ 350 \mathrm{~K}$

FOV: $>6^{\circ} @ \mathrm{HP}$

IFOV: $<2 \mathrm{mrad} @ \mathrm{HP}$

NETD: $<0.5 \mathrm{~K} @ 350 \mathrm{~K}$

$\Delta \mathrm{T}:<5 \mathrm{~K} @ 350 \mathrm{~K}$

Shape model: $10 \mathrm{~m}$ res.

Surface condition and materials (grain size, porosity, composition)

N.A.

Global thermal mapping:

- $\mathrm{T}$ profile by rotation

(accuracy <5 K)

- different solar distance

- spatial resolution $<20 \mathrm{~m}$

Global shape model ( $<10 \mathrm{~m}$ res.)

Rotation change ( $\sim^{\circ} /$ year $)$,

Orbit change (range $<\mathrm{km}$ )

Temperature distribution on boulder or base rock

- T difference between fresh surface on crater center to the surrounding area with spatial res. $<1 \mathrm{~m}$.
FOV: $>6^{\circ} @ \mathrm{HP}$

IFOV: $<2 \mathrm{mrad} @ \mathrm{HP}$

NETD: <0.5 K @350 K

$\Delta \mathrm{T}:<5 \mathrm{~K} @ 350 \mathrm{~K}$

Shape model: $10 \mathrm{~m}$ res.

Rotation detected by ONC $\left(<1^{\circ}\right)$

Range ( $<$ a few km)

FOV: $>6^{\circ} @ 1 \mathrm{~km}$

IFOV: $<1 \mathrm{mrad}$

NETD: $<0.5 \mathrm{~K} @ 350 \mathrm{~K}$

$\Delta \mathrm{T}:<5 \mathrm{~K} @ 350 \mathrm{~K}$ 
Table 5 (Continued)

Objectives Requirements for TIR

observation
Requirements for TIR

specification
Thermal modeling: topographic and roughness effects

- self-heating effects

- solar phase angle effect by grain size

SCI impact crater observation - Difference from surface to interior

- Area and grain size of ejecta

Surface geology and shape modeling

(even in the night side)

for $I>50$.

- Contemporary to optical

shape model

Dust measurement

- Dust environments around asteroid

- Dust ejection by SCI impact

Search for moons

- Existence of moons around small body

- Gravity information
Global thermal mapping and local site high res. thermal imaging

- T profile by rotation $(<5 \mathrm{~K})$

- Data at different solar distance

- Spatial res.: $<1 \mathrm{~m}$

Global shape model ( $<10 \mathrm{~m}$ res.)

Local surface model ( $<10 \mathrm{~cm}$ res.)

Surface conditions informed by

ONC, MINERVA, MASCOT, sample

Local high res. Thermal imaging

- Inside and surrounding area

of the SCI crater

- $\mathrm{T}$ profile by rotation $(<5 \mathrm{~K})$

- Global thermal mapping:

- $\mathrm{T}$ profile by rotation $(<5 \mathrm{~K})$

- Multiple solar distance

- Spatial res.: $<20 \mathrm{~m}$

- Wide angle ( $>$ a few asteroid diameter)

- Long time exposure in total (several seconds)

- Wide angle ( > a few asteroid diameter)

- Size down to $10 \mathrm{~m}$ class $(1 / 100$ (mass: $10^{-6}$ )
FOV: $>6^{\circ} @ 1 \mathrm{~km}$

IFOV: $<1 \mathrm{mrad}$

NETD: <0.5 K @350 K

$\Delta \mathrm{T}:<5 \mathrm{~K} @ 350 \mathrm{~K}$

Shape model: $10 \mathrm{~m}$ res.

Surface condition and materials (grain size, porosity, composition)

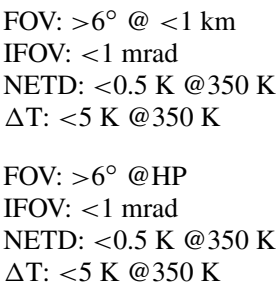

FOV: $>12^{\circ}$

Total integration: $>5 \mathrm{~s}$

FOV: $>12^{\circ} @ \mathrm{HP}(20 \mathrm{~km})$

IFOV: $<1 \mathrm{mrad}$

NETD: <0.5 K@350 K

$\Delta \mathrm{T}:<5 \mathrm{~K} @ 350 \mathrm{~K}$

\section{Description of the Thermal Infrared Imager TIR instrument}

\subsection{Outline of TIR}

TIR is a single-band thermal infrared imager with the wavelength ranging from 8 to $12 \mu \mathrm{m}$ and the field of view covering $16 \times 12^{\circ}$. The detector is based on a two-dimensional uncooled micro bolometer array (NEC 320A) with $328 \times 248$ effective pixels, a germanium detection window of anti-reflection coating, and a thermoelectric cooler module (Peltier module) to control the detector temperature at $313 \mathrm{~K}\left(40^{\circ} \mathrm{C}\right)$ within $0.1 \mathrm{~K}$ stability. The spatial resolution is about $0.05^{\circ}$ per pixel, which corresponds to $17 \mathrm{~m}$ per pixel when observing the asteroid from the Home Position at $20 \mathrm{~km}$ altitude. TIR has a shutter mechanism driven by a stepping motor and two positioning sensors. Images are taken in pairs, with the shutter closed and open respectively, and each pair of images thus consists of one dark image (shutter closed) and one exposed image (shutter open). The thermal images are then derived from the subtraction of the two images. The shutter temperature is monitored with $0.01 \mathrm{~K}$ accuracy, so that the bias of each pixel is cancelled. The imager has been well calibrated in the laboratory from $233 \mathrm{~K}$ to $423 \mathrm{~K}$, which covers the expected temperature range of the sunlit surface of asteroid Ryugu. The detectable temperature range is wider than this, from 

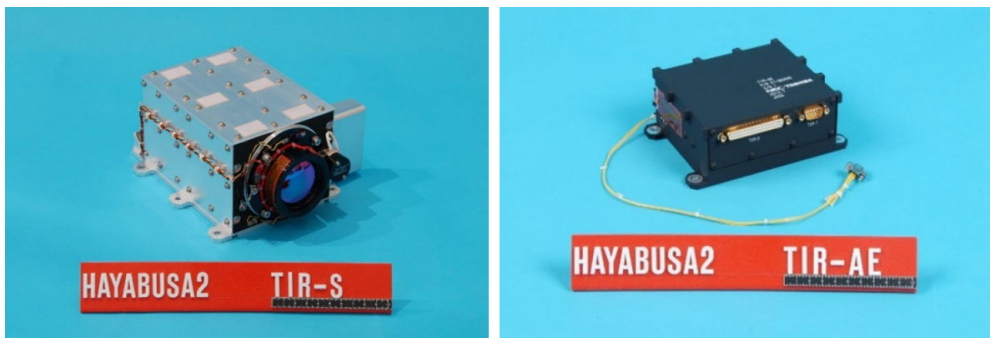

Fig. 4 Photographs of TIR-S (left) and TIR-AE (right)

Table 6 Performance of TIR

\begin{tabular}{ll}
\hline Mass & $3.28 \mathrm{~kg}$ \\
Power & $18 \mathrm{~W}$ (nominal) \\
Detector & Uncooled bolometer array NEC 320A (anti-reflection coating) \\
Pixels & $344 \times 260$ (effective $328 \times 248)$ \\
Field of view (FOV) & $16.7 \times 12.7^{\circ}$ \\
IFOV & $0.89 \mathrm{mrad}\left(0.051^{\circ}\right)$ \\
MTF (@Nyquist Freq.) & 0.5 \\
F-number & 1.4 \\
Temperature range & $233-423 \mathrm{~K}$ (well calibrated), 150-460 K (detectable range) \\
NETD & $<0.3$ \\
Absolute temperature range & $<3 \mathrm{~K}$ \\
A/D converter & 12 bit $(15$ bit after summed) \\
Reference temperature & Shutter temperature (monitored) \\
Frame rate & $1 / 60-2.1 \mathrm{~s}$ (summation for $m=1$ to 128 images) \\
\hline
\end{tabular}

$150 \mathrm{~K}$ to $460 \mathrm{~K}$, which covers the whole asteroid even in the nighttime for the thermal inertia $>50$ TI.

\subsection{Configuration and Functions of TIR}

TIR is a light-weighted thermal infrared imager, inherited from the Longwave Infrared Camera (LIR) onboard Akatsuki (PLANET-C) Venus climate orbiter (Fukuhara et al. 2011), which enabled development on a very short timescale and use of the same apparatus for calibration for the cold temperature range in the space chamber. TIR consists of the sensor unit (TIR-S), the power supply unit (TIR-AE), and the digital electronics unit (DE) (Hihara et al. 2014). The photographs of TIR-S and TIR-AE are shown in Fig. 4, and the performance of TIR is shown in Table 6. A 3D image of TIR-S is shown in Fig. 5.

TIR-S consists of two parts: the sensor body and the hood (sunshade). The sensor body includes the optics, detector, analog electronics, analog-to-digital converter, image processor, data interface to DE, telemetry and command interface, Peltier cooler controller, and power regulation units.

The optics unit has three pieces of Germanium lens, the mechanical shutter, and a band pass filter attached in the aperture. The diameters of lens and aperture are $47 \mathrm{~mm}$ and $25 \mathrm{~mm}$, respectively, and the $\mathrm{F}$ value is 1.4 . The filter passes from 8 to $12 \mu \mathrm{m}$. The mechanical shutter is located between the lens and filter, and operated using a stepping motor. The 
Fig. 5 The characteristic of TIR-S (modified from Fukuhara et al. 2011)
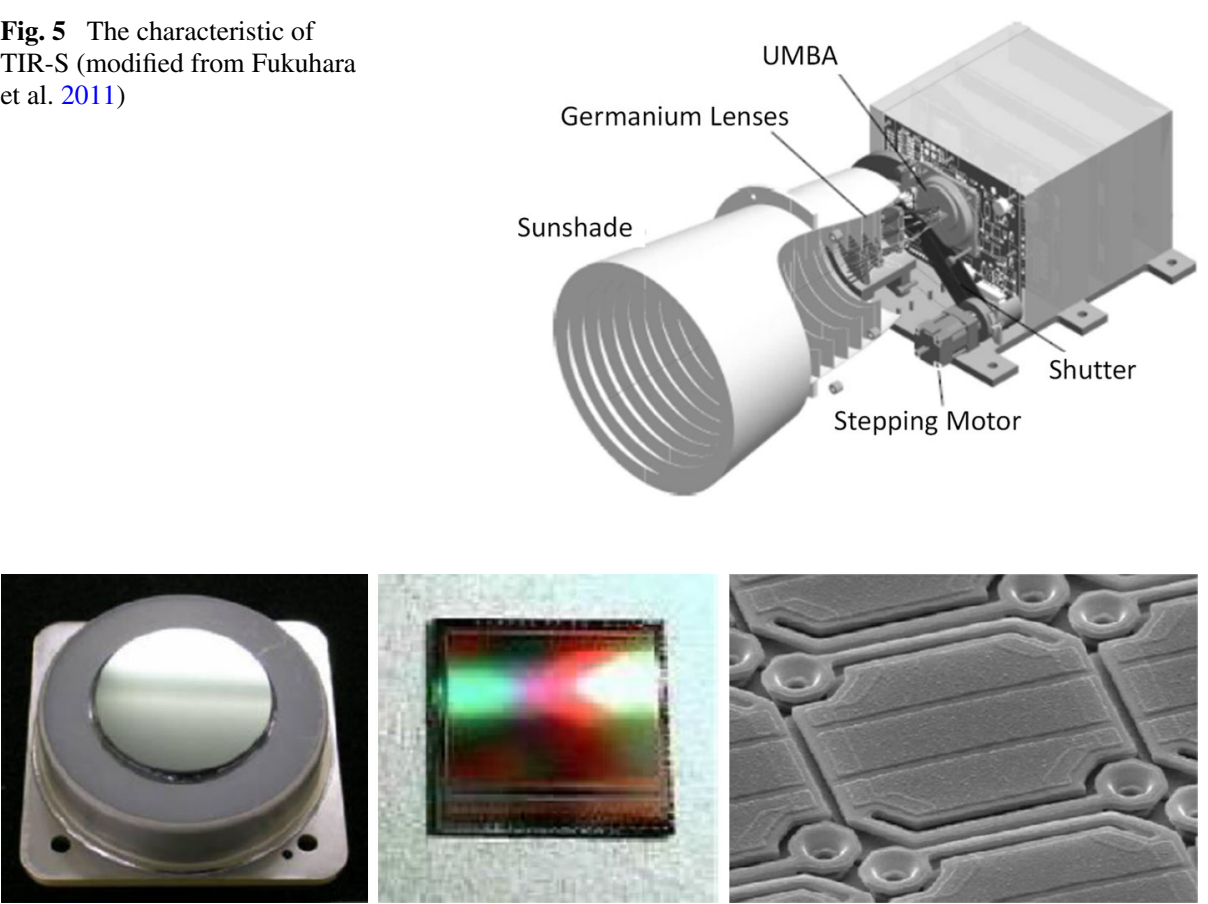

Fig. 6 Photograph of the detector package and the uncooled micro-bolometer array in the package, and scanning electron microscope image of a detector pixel (from Hihara et al. 2014)

shutter is only opened to take images and basically closed to protect direct irradiation of sunlight for contingency. The shutter is made of an aluminum plate with almite processing, or anodic oxidized coating, and its hemispherical emissivity is 0.89 . The temperature of the shutter is monitored at $0.01 \mathrm{~K}$ accuracy as Shutter Temperature, which is used as the reference temperature for calibration. The shutter position, i.e. whether it is open or closed, is detected with two position sensors. The filter is attached in the aperture, whose temperature is monitored at $0.01 \mathrm{~K}$ accuracy as Case Temperature. The optics unit is thermally controlled by the Heater Control Electronics (HCE) of the Hayabusa2 spacecraft. The temperature is monitored at $0.5 \mathrm{~K}$ accuracy with a sensor (Pt2000 resistance) as Channel 60A of HCE, and actually controlled in space within $0.3 \mathrm{~K}$ stability (monitored at $0.01 \mathrm{~K}$ accuracy as Lens Temperature in TIR-S), with proper settings of heater duty ratio and set points.

The detector unit NEC320A is originally a commercial package which includes the twodimensional bolometer array of $344 \times 260$ pixels and the temperature control system using a Peltier cooler. The detector has $328 \times 248$ effective pixels. Each pixel has $37 \times 37 \mu \mathrm{m}$ pitch and is $70 \%$ of the detection area. Six pixels at the top and bottom in the vertical axis are used as optical black pixels. Eight pixels at the left in the horizontal axis denote no output (noise level) and the next 8 pixels are used as optical black pixels. The cooler unit keeps the detector temperature at $313 \mathrm{~K}\left(40^{\circ} \mathrm{C}\right)$ or $283 \mathrm{~K}\left(10^{\circ} \mathrm{C}\right)$, selected by commands, and controls it within $0.01 \mathrm{~K}$ stability. Temperature stability is critical to TIR's output, and the main reason for TIR operating at $313 \mathrm{~K}\left(40^{\circ} \mathrm{C}\right)$ is its higher sensitivity at this temperature. The temperature of the detector package is also monitored at $0.01 \mathrm{~K}$ accuracy as Package Temperature. The detector unit and a close-up of a detector pixel are shown in Fig. 6. 


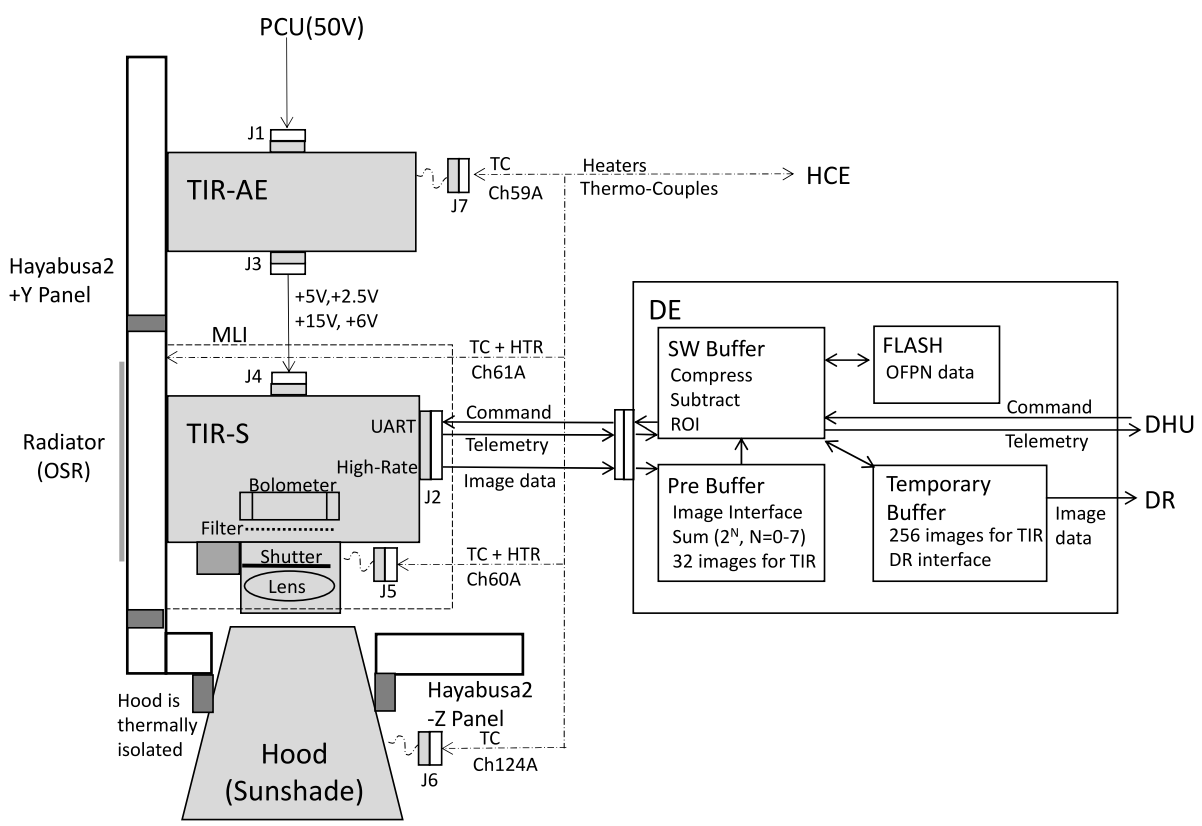

Fig. 7 Characteristic diagram of TIR-S, TIR-AE and DE interface

The hood is an aluminum-made horn-shaped sunshade to avoid direct input of sunlight. The length outside of $-Z$ panel is $150 \mathrm{~mm}$, with a $122-\mathrm{mm}$ diameter at the top. FOV of the hood is $22.92^{\circ}$ and the avoidance angle is $52^{\circ}$. The outside of the hood is effectively a radiator using $0.2 \mathrm{~mm}$ thick $\mathrm{Ag} /$ Teflon without ITO (Indium Tin Oxide) coating. Inside the hood there is a baffle structure with anodic oxidation coatings on aluminum. The hood is mainly used to prevent sunlight from warming the optics, but direct sunlight can cause damage to the bolometer pixels. The hood is thermally isolated from the $-Z$ panel of spacecraft. Temperature is monitored by HCE as Channel 124A.

TIR-AE is the power supply unit that converts the unregulated $50 \mathrm{~V}$ bus power to $+2.5 \mathrm{~V}$ and $+5 \mathrm{~V}$ for data processing, $+5 \mathrm{~V}$ for the detector, $+6 \mathrm{~V}$ for the Peltier cooler, and $+15 \mathrm{~V}$ for analog electronics. The temperature of TIR-AE is also monitored by HCE as Channel 59A.

DE is the digital electronics for DHU interface of all the scientific instruments and for the data recorder (DR). In addition, TIR uses the functions of DE such as the image data interface, image processing, image buffers (SDRAM $256 \mathrm{MB}$ ), and high-speed image data transfer to DR.

The characteristic diagram of the TIR and DE interface is shown in Fig. 7, and the diagram of signal flows and power lines in TIR-S and TIR-AE is shown in Fig. 8. Functions of each unit in TIR-S are summarized in Table 7, and details of functions related to TIR and related systems are shown in Table 8 .

\subsection{Imaging Processes of TIR}

TIR has six operation modes including Off, Standby, Protect, Idling, Parameter Setting, and Imaging modes. TIR can take an image only during the Idling mode. After the unregulated 


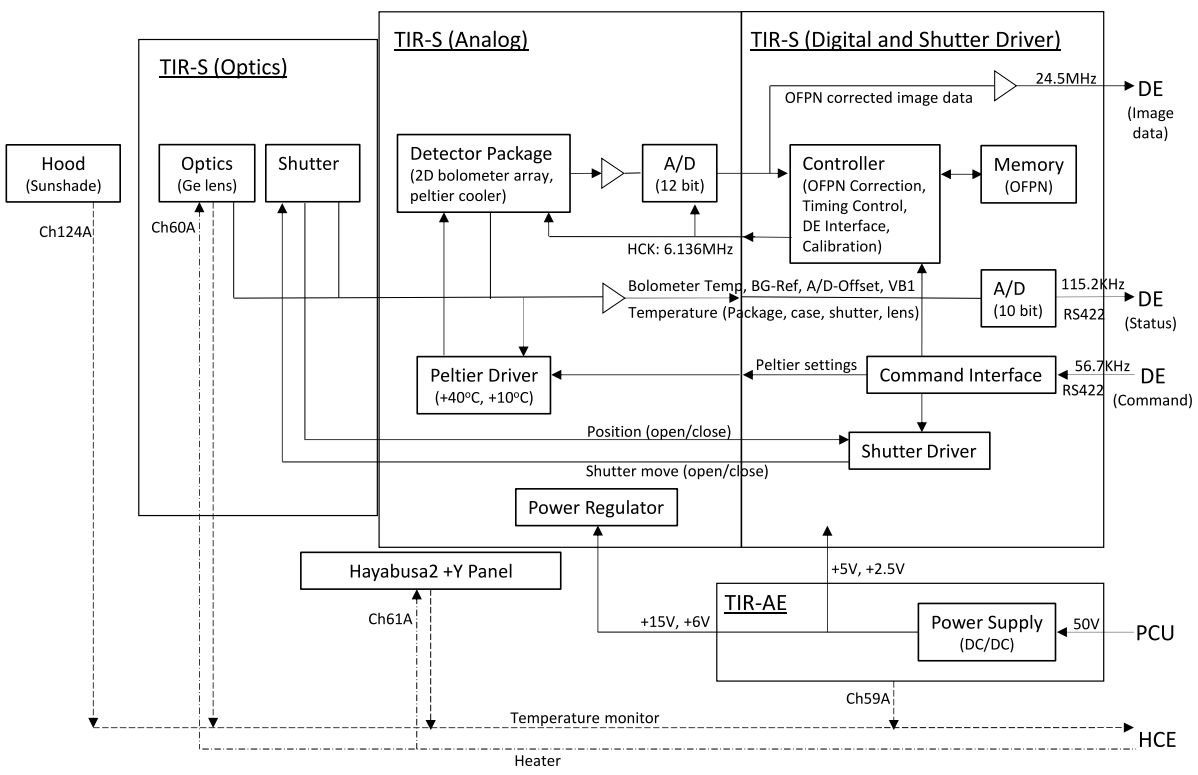

Fig. 8 Schematic diagram of signal and data flow, power lines, and temperature control lines in TIR-S and TIR-AE

Table 7 Summary of functions of each unit in TIR-S

\begin{tabular}{lll}
\hline Unit & Functions \\
\hline Optics & - & Configuration: Germanium lens (3 pieces): $\phi 47, \mathrm{~F} 1.4 / \mathrm{FL}=42.2 \mathrm{~mm}$, \\
& & MTF $>0.5$ \\
Shutter & - & Concentrate IR lights from outside to focus on the detector \\
& - & Band pass filter to limit the wavelength of $8-12 \mu \mathrm{m}$
\end{tabular}


Table 8 Functions of TIR

\begin{tabular}{|c|c|c|}
\hline Functions & Contents & Remarks \\
\hline $\begin{array}{l}\text { Command response } \\
\text { from DE }\end{array}$ & $\begin{array}{l}\text { Control and parameter settings for TIR by } \\
\text { commands. Maximum rate is } 1 / 16 \mathrm{~s}(62.5 \mathrm{~ms})\end{array}$ & UART interface \\
\hline Send telemetry to DE & $\begin{array}{l}\text { HK data and command response after commanded. } \\
\text { Every } 1 \text { second, and just before taking images }\end{array}$ & UART interface \\
\hline Imaging & Take images at 60 frames/sec, and A/D conversion & \\
\hline Image processing & $\begin{array}{l}\text { Calibration of images with the OFPN data and the } \\
\text { parameters set by commands }\end{array}$ & \\
\hline Send images to DE & Send the numbers of images set by commands & Dedicated line \\
\hline $\begin{array}{l}\text { OFPN data readout } \\
\text { and set }\end{array}$ & $\begin{array}{l}\text { Read the OFPN data from DE flash memory and set } \\
\text { into TIR-S memory }\end{array}$ & \\
\hline Get HK telemetry & $\begin{array}{l}\text { Get the latest HK data of TIR constantly and store } \\
\text { them into the register }\end{array}$ & \\
\hline $\begin{array}{l}\text { Bolometer } \\
\text { temperature control }\end{array}$ & $\begin{array}{l}\text { Control the bolometer temperature at } 40^{\circ} \mathrm{C} \text { or } 10^{\circ} \mathrm{C} \\
\text { by commands }\end{array}$ & Accuracy $< \pm 0.1 \mathrm{~K}$ \\
\hline Shutter open/close & $\begin{array}{l}\text { Dark image and Reference temperature. Fine/course } \\
\text { movement }\end{array}$ & Duration for $1 \mathrm{~s}$ \\
\hline Onboard calibration & Calibration onboard for bolometer outputs duration & Duration for $40 \mathrm{~s}$ \\
\hline Bolometer protection & Transfer to safe mode when the alert is detected & \\
\hline Sunshade & $\begin{array}{l}\text { Shield the direct sunshine or thermal emission off the } \\
\text { asteroid to avoid the lens too heated }\end{array}$ & $\begin{array}{l}\text { Avoidance angle } \\
\text { of the sunshade } \\
\text { is } \pm 26^{\circ}\end{array}$ \\
\hline $\begin{array}{l}\text { Regulation of input } \\
\text { current (DC/DC } \\
\text { converter) }\end{array}$ & $\begin{array}{l}\text { Regulate unregulated } 50 \mathrm{~V} \text { from PCU and supply } \\
+5 \mathrm{~V}(\mathrm{D}),+5 \mathrm{~V}(\mathrm{~A}),+2.5 \mathrm{~V},+6 \mathrm{~V},+15 \mathrm{~V}\end{array}$ & \\
\hline $\begin{array}{l}\text { Reference } \\
\text { temperature }\end{array}$ & $\begin{array}{l}\text { Monitor temperatures at Package, Case, Shutter, } \\
\text { Lens, and Hood }\end{array}$ & Accuracy $< \pm 0.1 \mathrm{~K}$ \\
\hline Image summation & $\begin{array}{l}\text { Sum the preset numbers of images at Pre-buffer of } \\
\text { DE, by } 2^{M}(M=0-7) \text {, stored as } 32 \text { bit data. Stored } \\
\text { address can be set for each image (max. } 32 \text { for TIR) }\end{array}$ & DE function \\
\hline Image subtraction & $\begin{array}{l}\text { Subtract between images stored in the buffer. } \\
\text { Thermal image is derived by shutter-open image } \\
\text { minus shutter-close one }\end{array}$ & DE function \\
\hline $\begin{array}{l}\text { Image summation by } \\
\text { SW }\end{array}$ & $\begin{array}{l}\text { Add, Subtract, multiply, divide images in the buffer. } \\
\text { MEAN images for } N=3 \sim 16 \text { images }\end{array}$ & DE function \\
\hline Image compression & $\begin{array}{l}\text { Compress images with StarPixel (lossless, lossy). } \\
\text { ROI (region of interest) can be set. }\end{array}$ & DE function \\
\hline $\begin{array}{l}\text { Temperature control } \\
\text { at lens }\end{array}$ & $\begin{array}{l}\text { Lens temperature can be controlled by } 0.5 \mathrm{~K} \text { step } \\
\text { by HCE }\end{array}$ & HCE function \\
\hline $\begin{array}{l}\text { Temperature control } \\
\text { at TIR-S mounted } \\
\text { panel }\end{array}$ & $\begin{array}{l}\text { Panel temperature can be controlled by } 0.5 \mathrm{~K} \text { step } \\
\text { by HCE }\end{array}$ & HCE function \\
\hline Monitor temperature & Monitor the temperature of Sunshade (Hood) & HCE function \\
\hline
\end{tabular}


Table 9 TIR operation mode

\begin{tabular}{lll}
\hline Modes & & Contents \\
\hline TIR & Off & Power off \\
& Standby & Initial state (peltier control = off) \\
& Protect & Safe mode for detector (peltier control = on) \\
& Idling & Ready for imaging \\
& Parameter & Detector internal correction and calibration \\
setting & \\
Imaging & Image taking and Data output from TIR \\
\hline
\end{tabular}

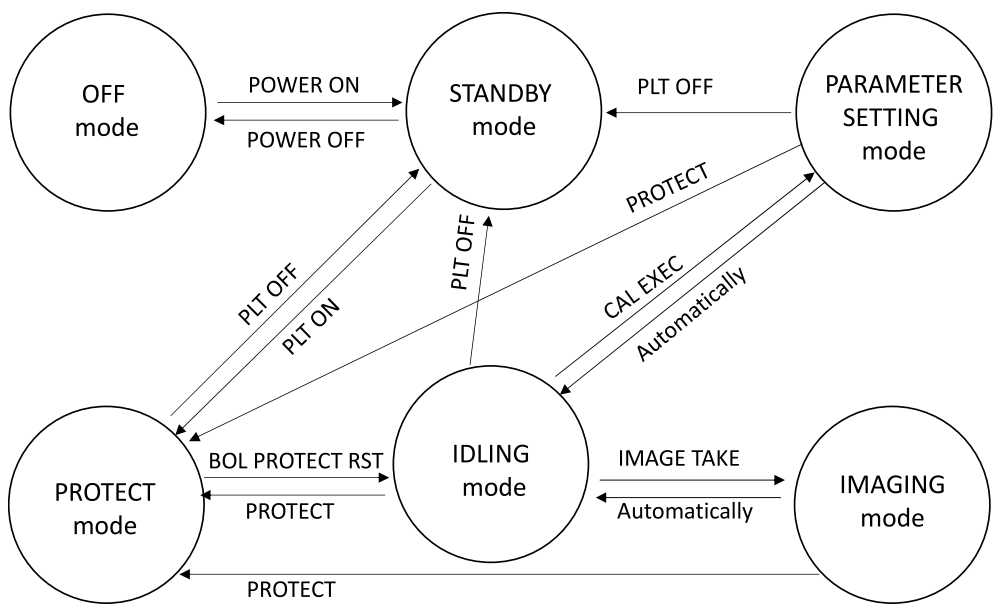

Fig. 9 Diagram of TIR mode transfer

$50 \mathrm{~V}$ power is supplied from PCU to TIR-AE, TIR starts and enters Standby mode. The Peltier cooler must be activated by sending the PLT ON command, and TIR enters Protect mode after the temperature of the bolometer is controlled at $313 \mathrm{~K}\left(40^{\circ} \mathrm{C}\right)$. Then TIR moves to Idling mode by sending BOL PROTECT RST command. The onboard flat noise pattern must be set in the TIR memory before taking an image. One method is to conduct onboard calibration by CAL EXEC command. Another method is to upload the OFPN data from DE, which is constructed on ground and uploaded beforehand. The TIR image is taken by sending IMAGE TAKE command. After the image has been taken, TIR automatically moves to Idling mode again. The definition of each mode is shown in Table 9, while the mode transfer-by-commands are shown in Fig. 9.

The signal readout values from the two-dimensional bolometer array have a large diversity pixel to pixel. Therefore TIR always takes a pair of images when the shutter is closed and open as mentioned in Sect. 4.1, corresponding to a dark frame image and an exposed one, respectively, and then subtracts the dark frame image from the exposed one. The shutter is basically closed during the mission, so that the first image is usually taken when the shutter is closed. The shutter has the quasi black body surface colored black by anodic oxidation coatings with an emissivity $\sim 0.89$. Its temperature is kept at $300 \mathrm{~K}$ to $303 \mathrm{~K}$ and monitored at the resolution of $0.01 \mathrm{~K}$. Thus the closed image can be used as the reference temperature for data analysis. The image is stored in the pre-buffer in the $1 \mathrm{MB}$ area of image interface 
in DE. The shutter is opened by the stepping motor in 1 second. Then the second image is taken when the shutter is open. The image is stored in the next $1 \mathrm{MB}$ area of the pre-buffer. There are two buffers with 128 MB SDRAM, and up to 128 pairs of TIR images can be taken and stored in the pre-buffer, but 32 images (16 pairs) are the maximum allocation for TIR and the rest are used for ONC in the current plan.

After taking images, the analog electronics unit reads the output signals from the detector at 60 frames per second and digitizes them into 12 bit image data through the analog-todigital converter. The readout data is compared with the onboard flat pattern noise (OFPN) for each pixel and produced as image data in the image processor in DE.

\subsection{Onboard Data Processing for TIR in DE}

The readout image data is transferred to the pre-buffer in the image data interface of DE. Before taking the image, the number of images to be summed can be set by commands. Currently $2^{N}$ images are summed there, where $N=0,1,2$, to, 7 . The original image digitized in TIR-S is 12-bit data but treated as 16-bit data in DE. In case of $N>4$, the image product becomes larger than 16 bit, so that the DE treats the TIR summed image as 32-bit data in the pre-buffer. The readout time, in case of $N=6$ (64 images to be summed), will take 64 images $\times 1 / 60$ second per frame $=64 / 60=1.07$ second for the summed image. The time delay between the images with closed and open shutter is typically 2 seconds.

After the image sequence finishes, DE transfers the summed images stored in the prebuffer to the software buffer, and then transfers them to the temporary buffer. Here only 16 bit data can be treated in the DE software buffer. The data compression algorithm used here is StartPixel (Hihara et al. 2014). The algorithm can only treat the image with 15-bit and $128 \times 128$ pixels. Thus, during this process, the images of $344 \times 260$ pixels $\times 32$-bit data in the pre-buffer are transformed to images of $384 \times 256$ pixels $\times 15$ bit, with 6 tiles of $128 \times 128$ pixels, and stored into the temporary buffer. In this procedure, four pixels at the bottom of the original image are erased, 40 pixels at the left are attached to the right, and all the data is bit-shifted to become 15-bit data as non-negative values in signed short format.

The pairs of images with shutter closed and open stored in the temporary buffer are subtracted to produce subtracted images. This operation is the equivalent of dark frame subtraction in conventional photometry. The subtracted images show good accuracy and stability is maintained quite well even if the temperature condition of TIR-S is changed. The subtracted images will be transferred to the data recorder (DR). Typically the image data is compressed using the StarPixel algorithm before transferring to DR.

Figure 10 shows examples of a TIR image pair of a $373 \mathrm{~K}$ blackbody target using the collimator (METS L-10-2.9, CI Systems) taken on 13 November 2013, and another TIR image pair of the deep sky in-flight observation taken on 27 February 2015. Image A is the shutter-open (exposed) image of the $373 \mathrm{~K}$ blackbody target and looks very noisy with dispersive bias pixel to pixel. Image B is the shutter-closed (dark frame) image, and Image C is derived by subtracting the two images, showing a clear blackbody target. The images D and $\mathrm{E}$ are a deep sky image (shutter open) and the corresponding dark frame image (shutter closed), respectively, while the image $\mathrm{F}$ is the result of subtraction of images D and $\mathrm{E}$.

\subsection{Observation Program}

In the Hayabusa2 mission, scientific operations are nominally conducted using an automatic program called Observation Program (OP, hereafter). An OP consists of the software se- 

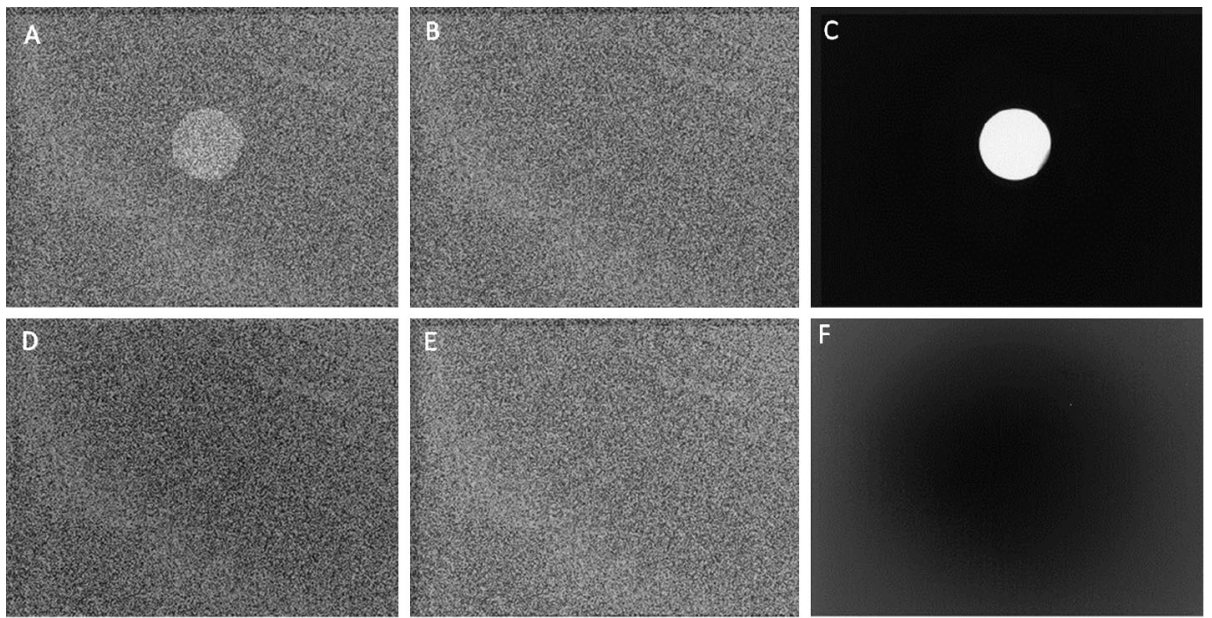

Fig. 10 Examples of TIR images are shown. Images of $373 \mathrm{~K}$ blackbody target using a collimator in front of TIR were taken in a pre-flight laboratory test on 13 November 2013 with shutter-open image (A), shutter-closed image $(\mathbf{B})$, and the subtracted image $(\mathbf{C})$. The deep sky images were taken on 27 February during the in-flight TIR with shutter-open image $(\mathbf{D})$, shutter-closed $(\mathbf{E})$, and the subtracted image $(\mathbf{F})$. The readout values have large diverse biases pixel to pixel, but subtraction of the shutter-closed from the shutter-open images results in low-noise thermal images. In image $\mathbf{F}$, TIR imaged the deep sky with an edge effect, and this is close to the background level of TIR

quences stored in DE, and 31 sequences can be stored at maximum. The sequences can be replaced by uploading new ones from the ground station. We have constructed and tested 17 OPs for TIR on the ground (see Table 10). At launch, only part of this program is stored in the DE because 31 OPs should be shared with all the instruments on Hayabusa2. The set of OPs should be changed before a new operation phase starts.

TIR- $0 \times 01$ is the setup sequence which is used every time TIR turns on. TIR- $0 \times 02$ conducts onboard calibration and take 4 images, which was mainly used for the system tests. This was also used for the first functionality test just after launch. TIR- $0 \times 03$ to $0 \times 05$ are for the function, performance, and health checks. TIR-06 to 08 take 128 summed images suitable for the observations at Home Position or during the cruise phase. TIR- $0 \times 09$ to $0 \times 0 \mathrm{C}$ take 32 summed images and are more suitable for the observations at lower altitude from the asteroid, since a 32-summed image takes 0.5 seconds per image, while it takes 2.1 seconds for a 128 -summed image. TIR- $0 \times 0 \mathrm{~B}$ is the special OP to track the SCI (Small Carry-on Impactor) continuously in a 2 -second interval (30 images) after its separation. TIR- $0 \times 0 \mathrm{D}$, shutter closed, is used for checking the shutter movement. TIR- $0 \times 0 \mathrm{E}-0 \mathrm{~F}$ are 128 - and 32 -summed images for four thermal images, but 1 of them is a full image and 3 of them are only the equatorial region to reduce the amount of data. TIR- $0 \times 10$ is the shutoff sequence of TIR to be used via OP. TIR- $0 \times 11$ is the sequence of 4 times TIR- $0 \times 0 \mathrm{E}$ at a 512 -second interval to be used during the descent phase of the Hayabusa 2 spacecraft. In Table 10, the TIR OPs are shown with their functions, duration, designated partitions of DR, and the amount of data production for each DR partition. The DR partition 69 is mainly used for subtracted images, $6 \mathrm{~A}$ is used for shutter-closed images, and $6 \mathrm{~F}$ is for the mean of subtracted images for quick downlink if required. 
Table 10 TIR operation program

\begin{tabular}{|c|c|c|c|c|c|}
\hline \# & Name of Obs program & Function & Duration [sec] & DR Pt. & Data $[\mathrm{MB}]$ \\
\hline TIR- $0 \times 01$ & $\begin{array}{l}\text { Setup and OFPN } \\
\text { loading }\end{array}$ & $\begin{array}{l}\text { Peltier ON, } \\
\text { Protect reset, } \\
\text { parameter settings, } \\
\text { OFPN data loading to } \\
\text { TIR }\end{array}$ & 500 & NA & NA \\
\hline TIR- $0 \times 02$ & Cal-Check & $\begin{array}{l}\text { Auto CAL without } \\
\text { OFPN, } \\
M=64 \text { image } \times 4 \text { set } \\
(\mathrm{C}, \mathrm{S}, \mathrm{A}) \\
\text { Image Compression }\end{array}$ & 128 & $\begin{array}{l}69 \\
6 \mathrm{~A} \\
6 \mathrm{~F}\end{array}$ & $\begin{array}{l}0.4 \\
0.8 \\
0.1\end{array}$ \\
\hline TIR- $0 \times 03$ & $\begin{array}{l}\text { Check1 (Function } \\
\text { check) }\end{array}$ & $\begin{array}{l}M=64 \text { image } \times 1 \text { set } \\
(\mathrm{C}, \mathrm{S}) \\
\text { Compressed }\end{array}$ & 64 & $\begin{array}{l}69 \\
6 \mathrm{~A}\end{array}$ & $\begin{array}{l}0.2 \\
0.4\end{array}$ \\
\hline TIR- $0 \times 04$ & $\begin{array}{l}\text { Check2 (Performance } \\
\text { check) }\end{array}$ & $\begin{array}{l}M=1,16,32,64, \\
128 \times 4 \text { set }(\mathrm{C}, \mathrm{S}, \mathrm{A}) \\
\text { Image compression }\end{array}$ & 360 & $\begin{array}{l}69 \\
6 \mathrm{~A} \\
6 \mathrm{~F}\end{array}$ & $\begin{array}{l}2.0 \\
4.0 \\
0.5\end{array}$ \\
\hline TIR- $0 \times 05$ & Check3 (Health check) & $\begin{array}{l}M=1,128 \times 16 \text { set } \\
(\mathrm{C}, \mathrm{S}, \mathrm{A}) \\
\text { Image compression }\end{array}$ & 600 & $\begin{array}{l}69 \\
6 \mathrm{~A} \\
6 \mathrm{~F}\end{array}$ & $\begin{array}{l}3.2 \\
6.4 \\
0.5\end{array}$ \\
\hline TIR- $0 \times 06$ & $\begin{array}{l}\text { Take1 (Nominal } \\
\text { exposure) }\end{array}$ & $\begin{array}{l}M=128 / N=4 \times 1 \text { set } \\
(\mathrm{C}, \mathrm{S}) \\
\text { Image compression }\end{array}$ & 64 & $\begin{array}{l}69 \\
6 \mathrm{~A}\end{array}$ & $\begin{array}{l}0.1 \\
0.2\end{array}$ \\
\hline TIR- $0 \times 07$ & Take 2 (Long exposure) & $\begin{array}{l}M=128 \times 16 \text { set } \\
(\mathrm{C}, \mathrm{S}, \mathrm{A}) \\
\text { Image compression }\end{array}$ & 352 & $\begin{array}{l}69 \\
6 \mathrm{~A} \\
6 \mathrm{~F}\end{array}$ & $\begin{array}{l}1.6 \\
3.2 \\
0.1\end{array}$ \\
\hline TIR- $0 \times 08$ & $\begin{array}{l}\text { Take3 (Short } \\
\text { exposure) }\end{array}$ & $\begin{array}{l}M=128 \times 1 \text { set }(\mathrm{C}, \mathrm{S}) \\
\text { Image compression }\end{array}$ & 32 & $\begin{array}{l}69 \\
6 \mathrm{~A}\end{array}$ & $\begin{array}{l}0.1 \\
0.2\end{array}$ \\
\hline TIR- $0 \times 09$ & Take6 (Moving object) & $\begin{array}{l}M=32 \times 16 \text { set } \\
(\mathrm{C}, \mathrm{S}, \mathrm{A}) \\
\text { Image compression }\end{array}$ & 288 & $\begin{array}{l}69 \\
6 \mathrm{~A} \\
6 \mathrm{~F}\end{array}$ & $\begin{array}{l}1.6 \\
3.2 \\
0.1\end{array}$ \\
\hline TIR- $0 \times 0 \mathrm{~A}$ & Take5 (Moving object) & $\begin{array}{l}M=32 \times 4 \text { set }(\mathrm{C}, \mathrm{S}, \mathrm{A}) \\
\text { Image compression }\end{array}$ & 96 & $\begin{array}{l}69 \\
6 \mathrm{~A} \\
6 \mathrm{~F}\end{array}$ & $\begin{array}{l}0.4 \\
0.8 \\
0.1\end{array}$ \\
\hline TIR- $0 \times 0 \mathrm{~B}$ & Take7 (SCI tracking) & $\begin{array}{l}M=16, \times 1(\mathrm{C}) \\
30(\mathrm{~S}), 1(\mathrm{C}) \\
\text { Image compression }\end{array}$ & 384 & $\begin{array}{l}69 \\
6 \mathrm{~A}\end{array}$ & $\begin{array}{l}3.0 \\
0.4\end{array}$ \\
\hline TIR- $0 \times 0 \mathrm{C}$ & Take4 (Close-up) & $\begin{array}{l}M=32 \times 1 \text { set }(\mathrm{C}, \mathrm{S}) \\
\text { Image compression }\end{array}$ & 32 & $\begin{array}{l}69 \\
6 \mathrm{~A}\end{array}$ & $\begin{array}{l}0.1 \\
0.2\end{array}$ \\
\hline TIR- $0 \times 0 \mathrm{D}$ & Shutter close & Shutter close for safe & 32 & NA & NA \\
\hline TIR- $0 \times 0 \mathrm{E}$ & $\begin{array}{l}\text { Take8 (HP } \\
\text { observations) }\end{array}$ & $\begin{array}{l}M=128 \times 4 \text { set }(\mathrm{C}, \mathrm{S}) \\
1 \text { for full, } 3 \text { for eq. only } \\
\text { Image compression }\end{array}$ & 96 & $\begin{array}{l}69 \\
6 \mathrm{~A}\end{array}$ & $\begin{array}{l}0.13 \\
0.8\end{array}$ \\
\hline TIR- $0 \times 0 \mathrm{~F}$ & Take9 (Low altitude) & $\begin{array}{l}M=32 \times 4 \operatorname{set}(\mathrm{C}, \mathrm{S}) \\
1 \text { for full, } 3 \text { for eq. only } \\
\text { Image compression }\end{array}$ & 96 & $\begin{array}{l}69 \\
6 \mathrm{~A}\end{array}$ & $\begin{array}{l}0.13 \\
0.8\end{array}$ \\
\hline TIR- $0 \times 10$ & Shut-off TIR & Shutoff sequence & 6 & NA & NA \\
\hline TIR- $0 \times 11$ & $\begin{array}{l}\text { OBS Sequence } \\
\text { (Descent) }\end{array}$ & $\begin{array}{l}4 \times \text { TIR }-0 \times 0 \text { E per } 512 \mathrm{~s} \\
\text { Image compression }\end{array}$ & 1532 & $\begin{array}{l}69 \\
6 \mathrm{~A}\end{array}$ & $\begin{array}{l}0.52 \\
3.2\end{array}$ \\
\hline
\end{tabular}



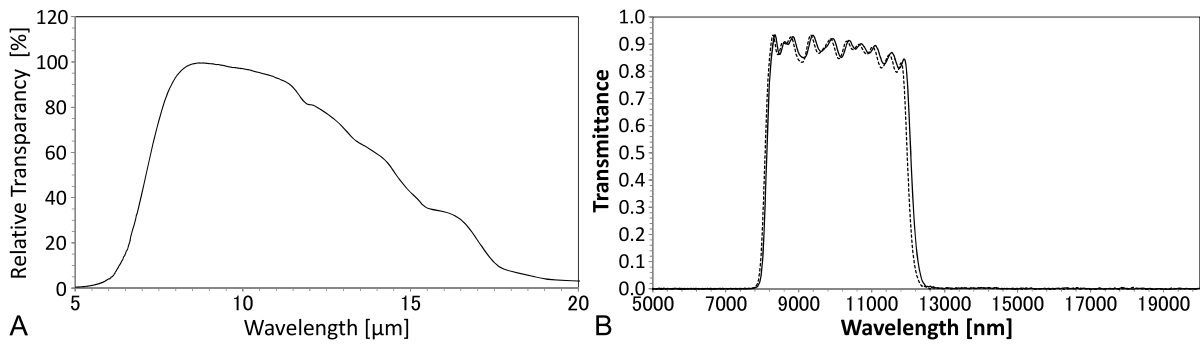

Fig. 11 The detection transparency of TIR is shown. (A) The combined transparency of 3 pieces of germanium lens relative to the peak at $8.5 \mu \mathrm{m}$ is shown as the function of wavelength. (B) Transparency function of the band-pass filter is shown for different angular conditions. The solid line shows the angle of incidence (aoi) $=0^{\circ}$ and half cone angle $(\mathrm{HCA})=7^{\circ}$, while the dashed line denotes the transparency for aoi $=16.4^{\circ}$ and $\mathrm{HCA}=21^{\circ}$

\section{Performance of TIR}

\subsection{Wavelength Range}

The TIR detection wavelength is determined by the combined transparency of 3 pieces of germanium lens, the detector package, as well as that of the band-pass filter mounted in front of the detector. Figure 11A shows the relative transparency of the germanium lens from 5 to $20 \mu \mathrm{m}$ with respect to transparency at $8.5 \mu \mathrm{m}$. Relative transparency is higher than $80 \%$ from 8 to $12 \mu \mathrm{m}$, although there is a tendency towards lower transparency for longer wavelengths. The commercial-based detector package is specifically designed for the $8-12 \mu \mathrm{m}$ band.

The transparency function of the band-pass filter is almost of rectangular shape, with sharp cut-offs on either side of the 7.9-12.1 $\mu \mathrm{m}$ band. In Fig. 11B, the solid line denotes the transparency spectrum for angle of incidence (aoi) $=0^{\circ}$ and half cone angle (HCA) $=7^{\circ}$, while the dashed line shows the transparency spectrum for aoi $=16.4^{\circ}$ and $\mathrm{HCA}=21^{\circ}$. Both profiles are almost the same, and the angular dependency is very small. This filter pattern is also considered for one of the filters on MARA on MASCOT (Helbert et al. 2014; Grott et al. 2016).

\subsection{Fields of View and Image Distortion}

Fields of view and image distortion of TIR are estimated using landscape images. In the attitude control test room in the ISAS satellite center (Building-C), TIR observed the geometric structures inside the room on 7 February 2014. One of the TIR images is shown in Fig. 12, a non-summed image using the DE simulator device. In this case, the dimensions of the geometric structures are known. The distance between each vertical pole of the veranda, $\mathrm{W}$, is $0.900 \mathrm{~m}$, which corresponds to $48 \pm 1$ pixels. Here we took the $4 \mathrm{~W}$ distance, corresponding to $194 \pm 1$ pixels. The distance from the position of the lens of TIR to the structures was measured with a portable laser range finder as $20.782 \pm 0.001 \mathrm{~m}$. The angular resolution of TIR or IFOV is derived as $0.018557 \pm 0.000096 \mathrm{~m} / \mathrm{pixel}$ around the central area of a TIR image, which corresponds to $0.891 \pm 0.009 \mathrm{mrad} / \mathrm{pixel}$ or $0.051 \pm 0.001^{\circ} / \mathrm{pixel}$.

The image distortion of TIR is verified by checking the linearity of each linear structure of geometric features and the parallelism between linear structures in this image. All the handrails in the horizontal direction show a good linearity within 1 pixel uncertainty for a length more than 250 pixels (examples are shown as the four dashed white lines in Fig. 12). 
Fig. 12 TIR image of geometric structures in the test facility. The dimensions of those structures are known so that IFOV and FOV is determined using the distance from TIR measured with a laser range finder. The image distortion is also verified as negligibly small using the parallelism of handrails (four dashed white horizontal lines) at a variety of positions in this image

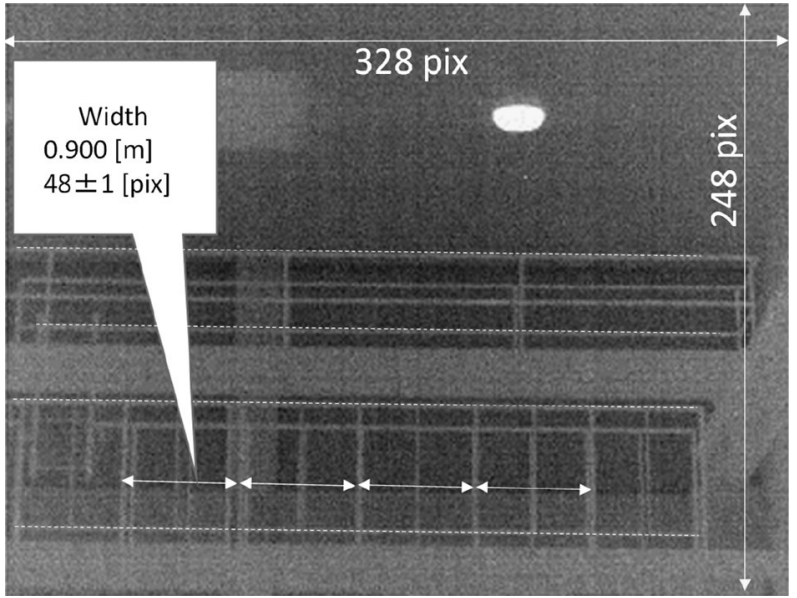

\begin{tabular}{ll}
\hline IFOV & $0.891 \pm 0.009 \mathrm{mrad} /$ pixel or $0.051 \pm 0.001^{\circ} /$ pixel \\
FOV & $16.74 \pm 0.17^{\circ} \times 12.66 \pm 0.13^{\circ}$ \\
\hline
\end{tabular}

Table 11 IFOV and FOV of TIR

The parallelism between handrails is also complete within 1 pixel uncertainty. The image distortion is also checked by the negligible difference between the distances of each vertical pole of the veranda. Thus we concluded that the image distortion is negligibly small. So the total FOV can be geometrically calculated as $16.74 \pm 0.17^{\circ}$ for the horizontal 328 pixels and $12.66 \pm 0.13^{\circ}$ for the vertical 248 pixels, respectively. Those results are summarized in Table 11.

The results of a more detailed geometrical calibration that was performed using the collimator set in the laboratory will be reported in future papers.

\subsection{Temperature Detection Range}

TIR's temperature detection range has been tested and calibrated in the laboratory, during system tests, and in space. Since the surface temperature of asteroid Ryugu is estimated to typically range from 250 to $400 \mathrm{~K}$ during daytime and down to $150 \mathrm{~K}$ during nighttime, it is difficult to calibrate the performance of radiation detectability of TIR using a single type of apparatus. Thus TIR was tested and calibrated with multiple apparatuses and test situations as shown in Table 12. The cross calibration between the apparatuses are needed and such work is in progress now. Detailed descriptions on calibration tests and the apparatuses will be reported in separate papers.

As for the cold target, the blackbody plate in the space chamber was selected because it is in a similar temperature region used for Akatsuki (Planet-C) mission calibration so that the same apparatus was used (Fukuhara et al. 2011). In this system, a blackbody target plate with homogeneous temperature (within $0.3 \mathrm{~K}$ ) is set in front of TIR-S and the entire field of view is covered by the target plate. The plate is monitored within $0.1 \mathrm{~K}$ accuracy at several points. This apparatus was originally built only for the cold temperature range, but by introducing an additional cooling system for TIR-S with silicone oil, the applicable temperature range was successfully extended to ambient temperature.

As a hot target, a commercial-based cavity blackbody was mainly used during the basic performance check. Note that there is a degree of uncertainty in the monitored temperature 
Table 12 The apparatus and their temperature ranges

\begin{tabular}{lll}
\hline $\begin{array}{l}\text { Test apparatus and test } \\
\text { situation }\end{array}$ & Temperature range & Remarks \\
\hline $\begin{array}{l}\text { Oil-bath blackbody } \\
\text { Cavity black body }\end{array}$ & 300 to $398 \mathrm{~K}$ & $\begin{array}{l}\text { Defocused, high stability, accuracy, } \\
\text { homogeneity } \\
\text { Defocused, easy to use, some uncertainty } \\
\text { in T }\end{array}$ \\
$\begin{array}{l}\text { Collimator with blackbody } \\
\text { with peltier control }\end{array}$ & 293 to $383 \mathrm{~K}$ & $\begin{array}{l}\text { Focused, High stability, geometrical } \\
\text { calibration }\end{array}$ \\
$\begin{array}{l}\text { Blackbody plate in space } \\
\text { chamber }\end{array}$ & 233 to $293 \mathrm{~K}$ & Defocused, uneasy for T control \\
$\begin{array}{l}\text { System thermal vacuum test } \\
\text { (IR panel) }\end{array}$ & 163 to $178 \mathrm{~K}$ & Defocused, rough temperature \\
$\begin{array}{l}\text { In-flight operation (during } \\
\text { cruise) }\end{array}$ & $<160 \mathrm{~K}$ & Deep sky survey \\
\hline
\end{tabular}

and the temperature homogeneity in the cavity. Next, an oil-bath blackbody apparatus was developed. The blackbody of this system shows a highly homogeneous temperature distribution and the temperature is well monitored within $0.1 \mathrm{~K}$ uncertainty from ambient to hot temperatures up to $423 \mathrm{~K}\left(150^{\circ} \mathrm{C}\right)$. However, as a result of the influence of thermal emission from the oil-bath, the maximum temperature was limited below $383 \mathrm{~K}\left(125^{\circ} \mathrm{C}\right)$ at that time. The oil-bath blackbody apparatus was also used for the cross-calibration of TIR with MARA on MASCOT. We also used the collimator system (IR System Co., METS L-10-2.9) but only for a limited number of temperatures, although the accuracy and stability of the blackbody temperature has been proven high enough. The collimator system was mainly used for geometrical calibration using patterned targets due to the limited time available until TIR delivery to the Hayabusa2 final test.

TIR's calibration was originally planned for observations of the sunlit region of asteroid Ryugu, but in order to confirm the noise level of the TIR-S, that is, the lowest temperature TIR can measure, we took some test situations to obtain data using lower temperature targets. The first one was during the system thermal vacuum test, where TIR-S viewed the black body IR panel about $1 \mathrm{~m}$ in front of TIR-S. The IR panel temperature was monitored at many points with $0.5 \mathrm{~K}$ accuracy, and we estimated the temperature of the IR panel with approximately $1 \mathrm{~K}$ uncertainty. The temperature ranged from $163 \mathrm{~K}$ to $178 \mathrm{~K}$ during the time TIR was tested using the pre-loaded OFPN data. However, at that time it was still unclear whether the lowest temperature measured at that time would be above the noise level.

After Hayabusa2 had been launched, TIR observed the deep sky, whose temperature should be lower than the detection level, and we regarded the data as the TIR-S system noise level. Indeed, we found that the values observed during deep sky observations were significantly lower than those for the IR panel during the system thermal vacuum test. Now we are able to conclude that the temperature at $160 \mathrm{~K}$ during the system vacuum test was effectively detected, and that the detection range is more likely to extend down to $150 \mathrm{~K}$.

Figure 13A shows the intensity for various temperatures taken by TIR. These values are taken from the average of $50 \times 50$ pixels in the central region of 128 frame-summed TIR images. As pointed out above, a single TIR image is 12-bit resolution but becomes 15-bit data after summation in the pre-buffer in DE and bit-shifted for software data processing in DE. In this graph the intensity is shown as 12-bit data, divided by 8 , for comparison with the non-summed single image data. We plot the deep sky at $100 \mathrm{~K}$, as we do not know its 

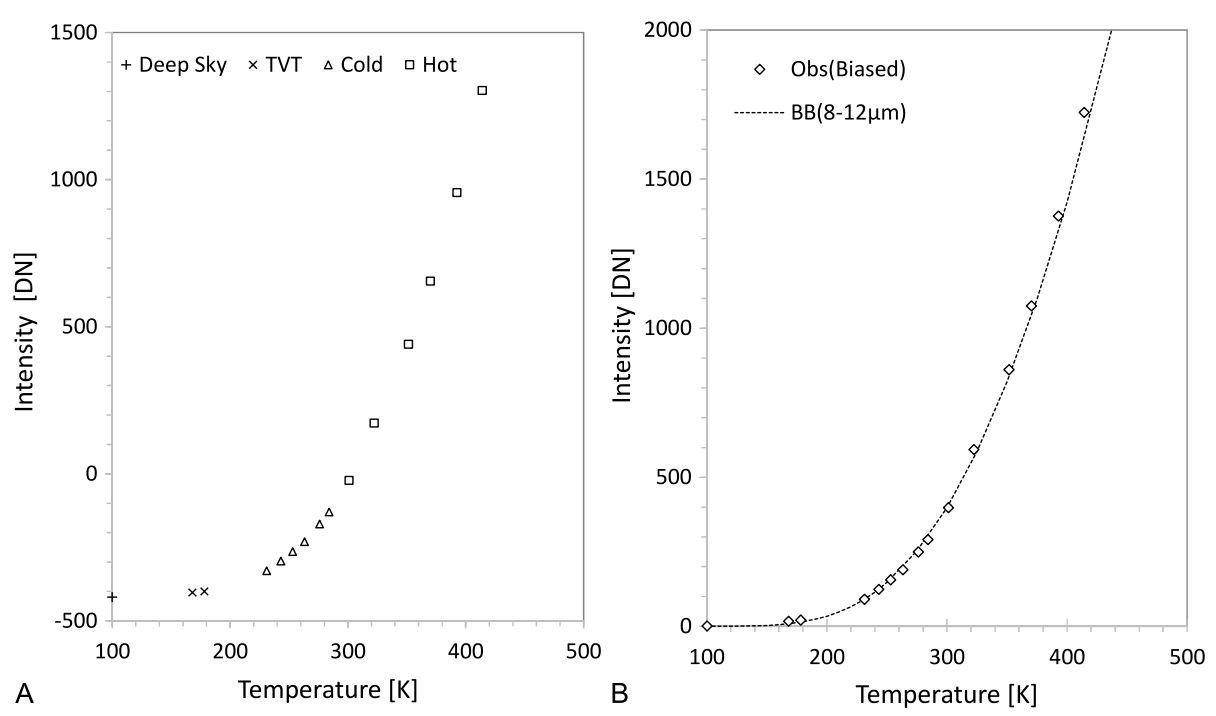

Fig. 13 Temperature detected by TIR using multiple apparatuses and opportunities. TIR calibration was conducted for Cold and Hot conditions using the apparatuses in the laboratory. Colder temperatures were detected during the system TVT test and the deep sky observations in space (A). A best fit line for the 8-12 $\mu \mathrm{m}$ range of blackbody radiation is shown along with the observed intensities which are biased by $421 \mathrm{DN}$ so that the biased intensity is set to 1 at $100 \mathrm{~K}(\mathbf{B})$

exact temperature which, nevertheless, can be expected to be lower than the IR panel in the system TVT test.

In Fig. 13B, we replot all the data with $421 \mathrm{DN}$ biased and also show the calculated relative intensity of blackbody radiation (Planck Function) in $8-12 \mu \mathrm{m}$ wavelength range. The biased intensity plots of TIR images and the calculated blackbody radiation fit well, at least for the temperature range of $230 \mathrm{~K}$ or higher. This fact implies that the thermal infrared detection of TIR is performed well and the system noise level is small ( $<\mathrm{a}$ few DN) to fit the well-calibrated range at $230 \mathrm{~K}$ or above.

The system noise level of TIR still remains somewhat uncertain, but examples of the system noise level of 1 or $10 \mathrm{DN}$ are shown in Fig. 14A and B, respectively, where the vertical axes are logarithmic. Vertical error bars of $\pm 3 \mathrm{DN}$ are shown in each plot because this is the typical dispersion of a 128-summed image. Apparently the intensities observed during the system TVT tests do not well fit with the blackbody radiation, but are consistent within the error bar.

\subsection{Temperature accuracy}

The absolute temperature accuracy of commercial thermal infrared imagers is typically 2 or $3 \mathrm{~K}$ due to the frame-to-frame dispersion of the bias. This is also the case for TIR when TIR takes a single image. The bias of all the pixels typically changes by several digits, corresponding to $3 \mathrm{~K}$ at maximum. We did not find a way to reduce this dispersion. Therefore it is practical to take multiple images. The sum of 128 images has a smaller dispersive bias offset than non-summed images. This is typically within $\pm 1 \mathrm{~K}$ for most of the temperature detection range. It is more practical to take several summed images. For this purpose, we prepare the OPs of TIR- $0 \times 0 \mathrm{E}$ and TIR- $0 \times 0 \mathrm{~F}$ with a full image and three equatorial region 

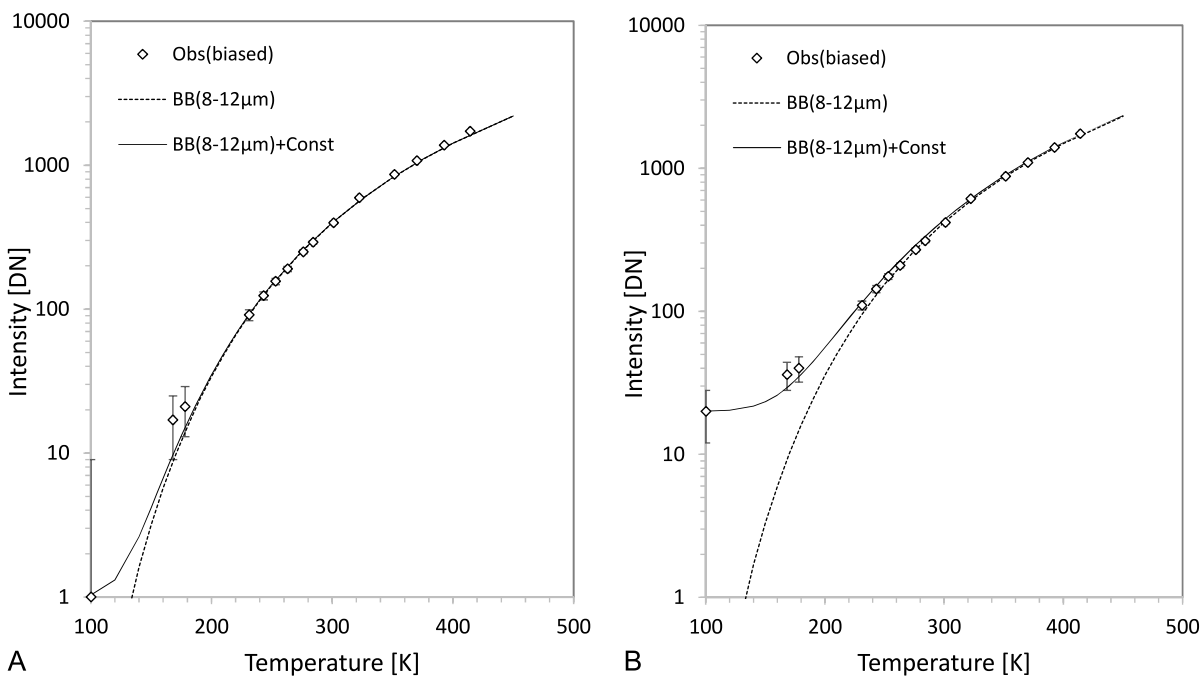

Fig. 14 The data shown in Fig. 13 plotted on a logarithmic scale, along with the blackbody radiation at $8-12 \mu \mathrm{m}$. The noise level is assumed for the case of $\mathbf{A}$ and $\mathbf{B}$, respectively

Fig. 15 NETD of the center area of TIR images, with open squares denoting the NETD derived from the relation of target temperature difference to the output value difference. The dashed line shows the calculated values assuming the values of TIR images are proportional to the band pass region of blackbody radiation energy and the noise level equals 1 digit level for non-summed images (or maximum 8 digits for summed images)

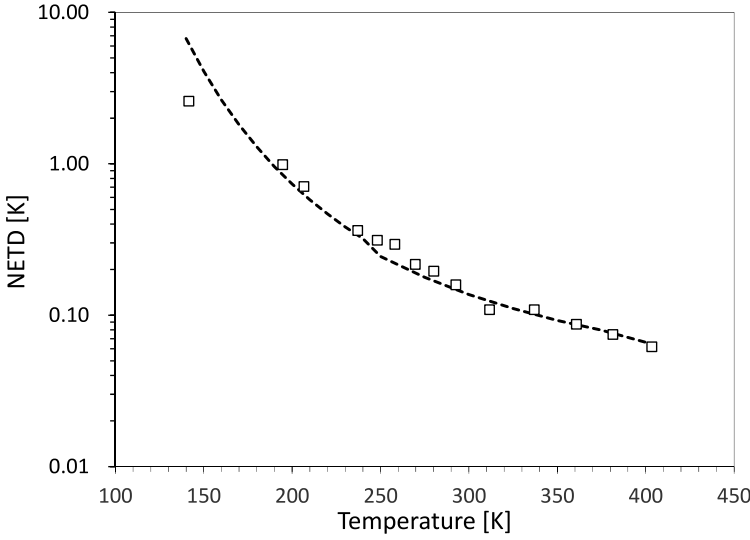

images of 128 and 32 summed images, respectively. The equatorial region image covers 16 instead of 256 vertical pixels only to check the dispersion of bias. The temperature dependence of the absolute temperature is basically compensated using the shutter temperature as reference and by monitoring the temperatures in TIR-S with $0.01 \mathrm{~K}$ accuracy.

In spite of the change of absolute temperature, the relative temperature in an image is not influenced by the bias change. Here, we take the relative temperature as noise-equivalent temperature difference (NETD) for the temperature dispersion at the same pixel in a time series. In this case the NETD must be defined for each pixel. Figure 15 shows the averaged values of NETD in the central region of TIR images as a function of target temperature. The open square mark denotes the NETD at the average data points plotted in Fig. 14, and the dashed line shows the calculated curves assuming that TIR values are proportional to the detection range ( 8 to $12 \mu \mathrm{m}$ band pass) of blackbody radiation (Planck equation). This result shows that the NETD is 0.3 or $0.4 \mathrm{~K}$ or less for $>230 \mathrm{~K}$, and the $1-\sigma$ noise level 
Table 13 Outline of TIR operation in Hayabusa2 mission

\begin{tabular}{|c|c|}
\hline Phase & Operation contents \\
\hline Launch & Shutter-close to avoid direct sunshine \\
\hline Initial operation & Check the functionality and basic performance after launch \\
\hline \multirow[t]{2}{*}{$\begin{array}{l}\text { Transfer and } \\
\text { EDVEGA }\end{array}$} & $\begin{array}{l}\text { Health and performance check by taking deep sky images } \\
\rightarrow \text { A long-term trend of degradation and a dependency of temperature }\end{array}$ \\
\hline & $\begin{array}{l}\text { Earth and Moon observations during Earth Swing-by } \\
\rightarrow \text { Alignment and radiometric check using Earth and Moon }\end{array}$ \\
\hline \multirow[t]{2}{*}{ Approach } & $\begin{array}{l}\text { Asteroid light-curve and radiometric measurements } \\
\rightarrow \text { Direct comparison with ground based observations }\end{array}$ \\
\hline & Moon search, and observations of environmental dust clouds \\
\hline \multirow[t]{8}{*}{ Rendezvous } & $\begin{array}{l}\mathrm{HP}(\sim 20 \mathrm{~km} \text { altitude): Global thermal imaging of asteroid } \\
\rightarrow \text { Construct a global thermo-physical model }(\sim 20 \mathrm{~m} / \text { pixel })\end{array}$ \\
\hline & $\begin{array}{l}\mathrm{XO}(\sim 20 \mathrm{~km} \text {, high phase angle): observations for polar and dawn-dusk regions } \\
\rightarrow \text { Complete the global thermo-physical model addition to HP observations }\end{array}$ \\
\hline & $\begin{array}{l}\text { MA }(\sim 5 \mathrm{~km} \text { mid altitude): Global TPM with higher resolution mapping }(3-5 \mathrm{~m} / \text { pixel }) \\
\rightarrow \text { Determine the TD site and geological context for the landed sites }\end{array}$ \\
\hline & $\begin{array}{l}\text { LA }(\sim 1 \mathrm{~km} \text { altitude): local site thermal imaging } \\
\rightarrow \text { TD and landers site context }(1 \mathrm{~m} / \mathrm{pixel})\end{array}$ \\
\hline & Close-up (10-1000 $\mathrm{m}$ altitude): highest resolution images during the descent \\
\hline & Landers landing site search after deploy (0.1-1 km altitude) \\
\hline & SCI tracking (500 $\mathrm{m}$ altitude): consecutive imaging of SCI \\
\hline & SCI crater search and observation ( $1 \mathrm{~km}$ or lower): SCI impact points \\
\hline
\end{tabular}

is about 1 digit for summed images. In the lower temperature range, the NETD becomes worse but remains within several K. A detailed calibration has been done, especially for the cross calibration between the apparatuses, so that more precise NETD will be reported in a separate paper both with regard to time series of each pixel and spatial region.

\section{Observation Plan of TIR}

\subsection{Outline of TIR Operation Plan}

The plan of TIR operations during the asteroid rendezvous phase is outlined in Table 13, and the schematic diagram is shown in Fig. 16. In order to avoid direct irradiation of sunshine into the TIR detector, TIR closed the shutter by command just after the separation of Hayabusa2 from the launch vehicle during the launch operation. During the initial function check on 11 December 2014, TIR checked its functionality using the quick check OP (TIR- $0 \times 02$ ), which was also used during the system environmental tests. For the performance test on 17 December 2014, TIR checked its performance using the health check OP (TIR- $0 \times 05$ ) with the temperature conditions adjusted using the HCE parameter settings by command. During the pre-flight test, we have learned that Shutter and Case temperatures should be stable as much as possible and set higher than $299.5 \mathrm{~K}$ and $300.0 \mathrm{~K}$, respectively. To achieve this condition, the Lens temperature (Channel 60A) and the Panel temperature (Channel 61A) both controlled by HCE should be adjusted at slightly higher setting points than those for the preservation ( $300.5 \mathrm{~K}$ and $298.0 \mathrm{~K}$, respectively). So far we find that setting points that are $1.5 \mathrm{~K}$ higher for both channels show good results. 
Fig. 16 The schematic diagram of the configuration of Hayabusa2 positions at the asteroid rendezvous phase is shown. Hayabusa2 will approach the asteroid from the sunward direction, mainly operated at the Home Position. The spacecraft will descend to the lower altitude for mission purposes

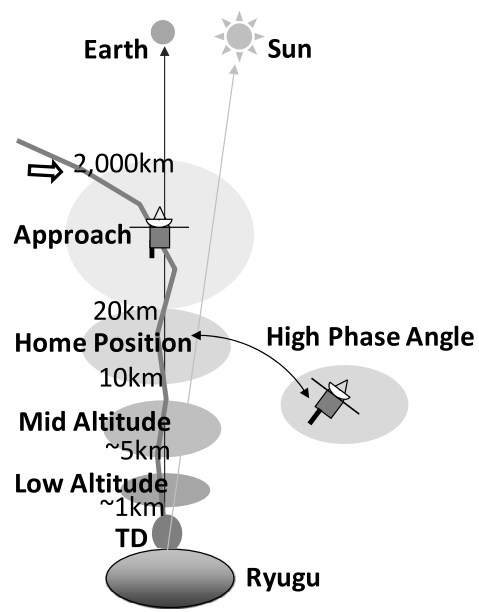

The health check will be continued once every month, and at the beginning of each operation phase. The observation plan will be presented in Sects. 6.2 to 6.7.

\subsection{Observation During Cruise and Earth Swing-By}

TIR will observe the deep sky once every month to conduct its health check using the health check OP (TIR- $0 \times 05$ ), to adjust the temperature conditions by HCE parameter settings, and to trace the possible degradation of the detector during the 3.5-year long cruise phase. The deep sky is considered a good target since it can be used as a kind of flat field cold target to estimate the noise level and check anisotropic response.

Just before and after the Earth swing-by, TIR is planned to image the Earth and the Moon using the health check OP (TIR- $0 \times 05$ ). One of the objectives is an alignment check with ONC and the attitude control of the Hayabusa2 spacecraft. When TIR observes the Earth and the Moon in an image concurrently with a wide angle camera (ONC-W1) or a telescopic camera (ONC-T), which is possible 30 to 10 days before the Earth swing-by, the alignment ( $-Z$ direction and roll angle of Hayabusa2 spacecraft) can be checked within a pixel accuracy. The other objectives of the observation are to measure the thermal radiation from the Earth or the Moon, since they are the only targets with known temperatures during the mission. Just after the Earth swing-by, TIR will observe the Earth and the Moon at closer distance using the health check OP $($ TIR- $0 \times 05)$ or low-altitude OPs $($ TIR- $0 \times 0 \mathrm{~A}$ and TIR- $0 \times 0 \mathrm{C})$.

\subsection{Observation whilst Approaching the Asteroid (AP)}

TIR will measure thermal infrared intensity and light-curves during the spacecraft's approach towards asteroid Ryugu and compare them with the results from ground-based observations. The asteroid is about the size of 1 pixel or less at a distance of $2000 \mathrm{~km}$ to $1000 \mathrm{~km}$ from the asteroid. We plan to take more than 20 images using the HP OP $($ TIR- $0 \times 0 \mathrm{E})$ during an asteroid rotation of 7.63 hours. After this phase, the asteroid becomes larger than 1 pixel so that we start observations not only for thermal emission but also for constructing shape models. At a distance of $100 \mathrm{~km}$ or closer to the asteroid, TIR will also observe the surrounding dust clouds and orbiting moons using the HP OP $($ TIR- $0 \times 0 \mathrm{E}$ ) or long exposure 
moving object OP $($ TIR- $0 \times 09)$. In case they are made of carbonaceous chondrite material, any moons are expected to be detected if larger than $1 / 5$ pixel in size. For example, this translates to $20 \mathrm{~m}$ object size for $100 \mathrm{~km}$ distance.

\subsection{Observations at Home Position (HP) and at Large Phase Angles (XO)}

After the arrival of the Home Position, about $20 \mathrm{~km}$ earthward from the asteroid, TIR will observe the asteroid once a week, by taking images using the HP OP (TIR- $0 \times 0 \mathrm{E})$ every 512 seconds during an asteroid rotation of 7.63 hours. More than 50 images are taken during one asteroid rotation. This is to be used to construct a thermal model with an improved thermal inertia compared to the one derived from a single thermal image. The thermal model of the whole asteroid will be constructed as the final product in this phase, with a spatial resolution of typically $17 \mathrm{~m}$ per pixel. A preliminary asteroid thermal model will be constructed sooner, probably in a week, for operational use. This model will be used to avoid hazardous operations by estimating the surface thermal environment during descent operations of the spacecraft, as well as assessing the distribution of boulders at the candidate sites on the asteroid.

A detailed asteroid thermal model constructed for scientific use will be available in a month, but updated according to updates of the asteroid shape model and the position and attitude of spacecraft. Due to the change of distance from the sun from 0.96 to $1.42 \mathrm{AU}$, the long-term trend of temperature change will be obtained and the thermal inertia resulting from thicker skin depths will be investigated. This model will be used for dedicated scientific studies and to constrain the surface particle size or possible existence of organics to select the landing sites of the mission.

Observations from large phase angles will also be planned. This phase is needed to map the entire surface completely and to obtain observations at different, higher phase angles. In particular for TIR, not only the polar region but the dawn and dusk regions could be easily observed. Since temperatures at the dawn and dusk regions are sensitive to the surface thermal inertia, as shown in Fig. 1, these observations are important to investigate thermal inertia.

\subsection{Lower Altitude Observations (MA, LA)}

Before the descent operations for sample collection or deployment of landing robots, the spacecraft will descend to a lower altitude and observe the candidate sites in more detail to confirm whether the sites are suitable with regard to scientific and mission requirements. One or two asteroid rotations (7.63 and 15.26 hours) before arrival at target altitude, TIR will image the surface using the descent OP (TIR- $0 \times 11)$. At $5 \mathrm{~km}$ altitude (MA), TIR will take images using the moving object OP (TIR- $0 \times 0 \mathrm{C}$ ) every 512 or 256 seconds and map the hemisphere including the candidate sites with $4.3 \mathrm{~m}$ spatial resolution. These images are used to confirm the thermal model constructed beforehand, and also to construct a more detailed thermal model.

At $1 \mathrm{~km}$ altitude (LA), TIR will take images of specific sites at $85 \mathrm{~cm}$ per pixel and $280 \times 210 \mathrm{~m}$ area, probably in an hour, using the moving object OP (TIR- $0 \times 0 \mathrm{C})$. Geologic features are imaged at proper intervals and investigated for their thermo-physical properties.

\subsection{Descent, Close-Up, and Touchdown Observations (CU, TD)}

For sample collection, or deployment of landing robots, as well as their rehearsals, the spacecraft will descend to a lower altitude. Again, 1 or 2 asteroid rotations ( 7.63 and 15.26 hours) 
before the arrival at the target sites, TIR will image the surface using the descent OP (TIR- $0 \times 11)$. At a lower altitude between $1 \mathrm{~km}$ and $50 \mathrm{~m}$, TIR will also observe the surface of the asteroid at a higher spatial resolution using the moving object OP (TIR- $0 \times 0 \mathrm{C}$ ) every 256 second nominally.

For the final descent from $50 \mathrm{~m}$ to $5 \mathrm{~m}$ altitude, TIR will image the surface using the moving object OP (TIR- $0 \times 0 \mathrm{C})$ more frequently, namely every 60 seconds. The final spatial resolution is an order of centimeters. TIR will stop its observations using the shutoff OP (TIR- $0 \times 10)$ at $5 \mathrm{~m}$ altitude just before the start of the free fall of the spacecraft for touchdown.

For the deployment of landing robots such as MINERVA-II and MASCOT, the altitude of deployment will be about $60 \mathrm{~m}$. TIR will image the landing sites using the moving object OP (TIR- $0 \times 0 \mathrm{C}$ ). After deployment, the spacecraft will ascend to $3 \mathrm{~km}$ altitude and hover at altitude for 24 hours, then ascend to HP. TIR will observe the asteroid surface using the moving object OP (TIR- $0 \times 0 \mathrm{C})$ every 512 seconds nominally.

\subsection{SCI Separation and SCI Search}

For the SCI separation, the spacecraft will descend to $500 \mathrm{~m}$ altitude, deploy SCI, and escape from there to the safe area behind the asteroid. Again, 1 or 2 asteroid rotations (7.63 and 15.26 hours) before the arrival of target sites, TIR will image the surface using the descent OP (TIR- $0 \times 11$ ). At the altitude of $1 \mathrm{~km}$ to $500 \mathrm{~m}$, TIR will image the surface using the moving object OP (TIR- $0 \times 0 \mathrm{C})$. Just after separation of SCI, TIR will take images of SCI using the SCI tracking OP (TIR- $0 \times 0 \mathrm{~B}), 30$ images for each 2 -second interval. After escape from there and behind the asteroid, TIR will observe the dust stream originating from the surface of asteroid excavated by the SCI impact, using the long exposure moving object OP (TIR-0×09).

\section{Summary}

TIR is the thermal infrared imager on Hayabusa2 designed to map the thermo-physical properties of C-type near-Earth asteroid 162173 Ryugu. We have demonstrated the scientific importance of thermal infrared imaging and presented the scientific and mission objectives. The specifications of TIR have been described, showing that TIR has a relatively wide field of view of $16 \times 12^{\circ}$ to image the thermal emission from the asteroid and also covers a wide temperature range of $150 \mathrm{~K}$ to $460 \mathrm{~K}$ with $230-420 \mathrm{~K}$ as the well-calibrated range. The performance meets scientific and system requirements. Detailed instrumentation and data processing flows have been shown. A detailed operation plan was also given for launch, cruise, home position and low-altitude operations. The thermal model obtained from TIR data will contribute to understanding the nature of the target asteroid and will also be valuable for sampling site selection and landing safely.

Acknowledgements We appreciate all the members of Hayabusa2 project for scientific and technical support for the development and operation of TIR. This research is partly supported by the Grant-in-Aid for Scientific Research (B), No. 26287108, of the Japan Society for the Promotion of Science.

Open Access This article is distributed under the terms of the Creative Commons Attribution 4.0 International License (http://creativecommons.org/licenses/by/4.0/), which permits unrestricted use, distribution, and reproduction in any medium, provided you give appropriate credit to the original author(s) and the source, provide a link to the Creative Commons license, and indicate if changes were made. 


\section{References}

J. Biele, S. Ulamec, M. Maibaum, R. Roll, L. Witte, E. Jurado, P. Muñoz, W. Arnold, H.-U. Auster, C. Casas, C. Faber, C. Fantinati, F. Finke, H.-H. Fischer, K. Geurts, C. Güttler, P. Heinisch, A. Herique, S. Hviid, G. Kargl, M. Knapmeyer, J. Knollenberg, W. Kofman, N. Kömle, E. Kührt, V. Lommatsch, S. Mottola, R. Pardo de Santayana, E. Remetean, F. Scholten, K. Seidensticker, H. Sierks, T. Spohn, The landing(s) of Philae and inferences about comet surface mechanical properties. Science 349, aaa9816 (2015)

W.F. Bottke Jr., D. Vokrouhlický, D.P. Rubincam, D. Nesvorný, The Yarkovsky and YORP effects: implications for asteroid dynamics. Annu. Rev. Earth Planet. Sci. 34, 157-191 (2006)

S.C. Chase, E. Minor, D. Morrison, G. Muench, G. Neugebauer, M. Schroeder, Mariner 10 infrared radiometer results: temperatures and thermal properties of the surface of Mercury. Icarus 28, 565-578 (1976)

P.R. Christensen, J. Bandfield, V. Hamilton, S. Ruff, H. Kieffer, T. Titus, M. Malin, R. Morris, M. Lane, R. Clark, B. Jakosky, M. Mellon, J. Pearl, B. Conrath, M. Smith, R. Clancy, R. Kuzmin, T. Roush, G. Mehall, N. Gorelick, K. Bender, K. Murray, S. Dason, E. Greene, S. Silverman, M. Greenfield, Mars Global Surveyor Thermal Emission Spectrometer Experiment: investigation description and surface science results. J. Geophys. Res. 106, 23,823-23,871 (2001)

P.R. Christensen, J. Bandfield, J. Bell III., N. Gorelick, V. Hamilton, A. Ivanov, B. Jakosky, H. Kieffer, M. Lane, M. Malin, T. McConnochie, A. McEwen, H. McSween Jr., G. Mehall, J. Moersch, K. Nealson, J. Rice Jr., M. Richardson, S. Ruff, M. Smith, T. Titus, M. Wyatt, Morphology and composition of the surface of Mars: Mars Odyssey THEMIS results. Science 300, 2056-2061 (2003)

G.J. Consolmagno, D. Britt, R. Macke, The significance of meteorite density and porosity. Chem. Erde 68, 1-29 (2008)

M. Delbo, A. dell'Oro, A. Harris, S. Mottola, M. Mueller, Thermal inertia of near-Earth asteroids and implications for the magnitude of the Yarkovsky effect. Icarus 190, 236-249 (2007)

A. Fujiwara, J. Kawaguchi, D.K. Yeomans, M. Abe, T. Mukai, T. Okada, J. Saito, H. Yano, M. Yoshikawa, D.J. Scheeres, O. Barnouin-Jha, A.F. Cheng, H. Demura, R.W. Gaskell, N. Hirata, H. Ikeda, T. Kominato, H. Miyamoto, A.M. Nakamura, R. Nakamura, S. Sasaki, K. Uesugi, The Rubble-Pile asteroid Itokawa as observed by Hayabusa. Science 312, 1330-1334 (2006)

T. Fukuhara, M. Taguchi, T. Imamura, M. Nakamura, M. Ueno, M. Suzuki, N. Iwagami, M. Sato, K. Mitsuyama, G.L. Hashimoto, R. Ohshima, T. Kouyama, H. Ando, M. Futaguchi, LIR: longwave infrared camera onboard the Venus orbiter Akatsuki. Earth Planets Space 63, 1009-1018 (2011)

M.P. Golombek, A. Haldemann, N. Forsberg-Taylor, E. DiMaggio, R. Schroeder, B. Jakosky, M. Mellon, J. Matijevic, Rock size-frequency distributions on Mars and implications for Mars Exploration Rover landing safety and operations. J. Geophys. Res. 108, 27-1-27-23 (2003). 2003. doi:10.1029/2002JE002035

M. Grott, J. Knollenberg, B. Borgs, F. Hänschke, E. Kessler, J. Helbert, A. Maturilli, N. Müller, The MASCOT radiometer MARA for the Hayabusa2 mission. Space Sci. Rev. (2016, this issue). doi:10.1007/s11214-016-0272-1

O. Groussin, J.M. Sunshine, L.M. Feaga, L. Jorda, P.C. Thomas, J.-Y. Li, M.F. A'Hearn, M.J.S. Belton, S. Besse, B. Carcich, T.L. Farnham, D. Hampton, K. Klaasen, C. Lisse, F. Merlin, S. Protopapa, The temperature, thermal inertia, roughness and color of the nuclei of comets 103P/Hartley 2 and 9P/Tempel 1. Icarus 222, 580-594 (2013)

J. Helbert, S. del Togno, A. Maturilli, S. Ferrari, M. Grott, B. Borgs, T. Okada, A novel spectral and radiometric calibration target for the TIR imager and the MARA instrument on the Hayabusa2 mission. Proc. Lunar Planet. Sci. Conf. 45, 1317 (2014)

H. Hiesinger, J. Helbert (MERTIS Co-I Team), The Mercury radiometer and thermal infrared spectrometer (MERTIS) for the BepiColombo mission. Planet. Space Sci. 58(1-2), 144-165 (2010)

H. Hihara, K. Iwase, J. Sano, H. Otake, T. Okada, R. Funase, R. Kashikawa, I. Higashino, T. Masuda, SpaceWire-based thermal-infrared imager system for asteroid sample return mission HAYABUSA2. J. Appl. Remote Sens. 8(1), 084987 (2014)

T.-M. Ho, V. Baturkin, R. Findlay, C. Grimm, J.T. Grundmann, C. Hobbie, E. Ksenik, C. Lange, K. Sasaki, M. Schlotterer, M. Talapina, N. Termtanasombat, E. Wejmo, L. Witte, M. Wrasmann, G. Wübbels, J. Rößler, C. Ziach, J. Biele, C. Krause, S. Ulamec, M. Lange, O. Mierheim, R. Lichtenheldt, M. Meier, J. Reill, H.-J. Sedlmayr, P.W. Bousquet, A. Bellion, O. Bompis, C. Cenac-Morthe, M. Deleuze, S. Fredon, E. Jurado, E. Canalias, R. Jaumann, J.-P. Bibring, K.-H. Glassmeier, M. Grott, L. Celotti, F. Cordero, J. Hendrikse, T. Okada, MASCOT-the mobile asteroid surface scout onboard the HAYABUSA2 mission. Space Sci. Rev. (2016, this issue). doi:10.1007/s11214-016-0251-6

M. Ishiguro, D. Kuroda, S. Hasegawa, M.-J. Kim, Y.-J. Choi, N. Moskovitz, S. Abe, K.-S. Pan, J. Takahashi, Y. Takagi, A. Arai, N. Tokimasa, H.H. Hsieh, J.E. Thomas-Osip, D.J. Osip, M. Abe, M. Yoshikawa, S. Urakawa, H. Hanayama, T. Sekiguchi, K. Wada, T. Sumi, P.J. Tristram, K. Furusawa, F. Abe, A. Fukui, T. Nagayama, D.S. Warjurkar, A. Rau, J. Greiner, P. Schady, F. Knust, F. Usui, T.G. Müller, 
Optical properties of (162173) 1999JU3: in preparation for the JAXA Hayabusa2 sample return mission. Astrophys. J. 792, 74 (2014), 9 pp.

T. Iwata, K. Kitazato, M. Abe, M. Ohtake, Ta. Arai, To. Arai, N. Hirata, T. Hiroi, C. Honda, N. Imae, M. Komatsu, T. Matsunaga, M. Matsuoka, S. Matsuura, T. Nakamura, A. Nakato, Y. Nakauchi, T. Osawa, H. Senshu, Y. Takagi, K. Tsumura, N. Takato, S. Watanabe, M.-A. Barucci, E. Palomba, M. Ozaki, NIRS3: the near infrared spectrometer on Hayabusa2. Space Sci. Rev. (2016, this issue)

H.H. Kieffer, S. Chase, E. Minor, G. Muench, G. Neugebauer, Preliminary report on infrared radiometric measurements from the Mariner 9 spacecraft. J. Geophys. Res. 78, 4291-4312 (1973)

H.H. Kieffer, T. Martin, A. Peterfreund, B. Jakosky, Thermal and albedo mapping of Mars during Viking primary mission. J. Geophys. Res. 82, 4249-4291 (1977)

P. Michel, M. Delbo, Orbital and thermal evolutions of four potential targets for a sample return space mission to a primitive near-Earth asteroid. Icarus 209, 520-534 (2010)

T.G. Müller, J.D. urech, S. Hasegawa, M. Abe, K. Kawakami, T. Kasuga, D. Kinoshita, D. Kuroda, S. Urakawa, S. Okumura, Y. Sarugaku, S. Miyasaka, Y. Takagi, P.R. Weissman, Y.-J. Choi, S. Larson, K. Yanagisawa, S. Nagayama, Thermo-physical properties of 162173 (1999 JU3), a potential flyby and rendezvous target for interplanetary missions. Astron. Astrophys. 525, A145 (2011). doi:10.1051/0004-6361/201015599

T. Okada, Overviews of Hayabusa2: the C-class asteroid sample return mission of Japan, in Proc. International CJMT-1 Workshop on Asteroid Science, Oct. 2012, Macau University of Science and Technology, ed. by W. Ip (2014), pp. 60-73. 2014

T. Okada, Thermal inertia of surface materials of solar system small bodies and its dependence on porosity. Proc. Lunar Planet. Sci. Conf. 47, \#1457 (2016)

D.A. Paige, M. Foote, B. Greenhagen, J. Schofield, S. Calcutt, A. Vasavada, D. Preston, F. Taylor, C. Allen, K. Snook, B. Jakosky, B. Murray, L. Soderblom, B. Jau, S. Loring, J. Bulharowski, N. Bowles, I. Thomas, M. Sullivan, C. Avis, E. De Jong, W. Hartford, D.J. McCleese, The Lunar Reconnaissance Orbiter Diviner Lunar Radiometer Experiment. Space Sci. Rev. (2009). doi:10.1007/s11214-009-9529-2

T. Spohn, J. Knollenberg, A.J. Ball, M. Banaszkiewicz, J. Benkhoff, M. Grott, J. Grygorczuk, C. Hüttig, A. Hagermann, G. Kargl, E. Kaufmann, N. Kömle, E. Kührt, K.J. Kossacki, W. Marczewski, I. Pelivan, K. Seiferlin, Thermal and mechanical properties of the near-surface layers of 67P/ChuryumovGerasimenko from in situ measurements at Abydos with the MUPUS instrument package. Science 349(6247), aab0464 (2015). doi:10.1126/science.aab0464

S. Tachibana, M. Abe, M. Arakawa, M. Fujimoto, Y. Iijima, M. Ishiguro, K. Kitazato, N. Kobayashi, N. Namiki, T. Okada, R. Okazaki, H. Sawada, S. Sugita, Y. Takano, S. Tanaka, S. Watanabe, M. Yoshikawa, H. Kuninaka, Hayabusa2: scientific importance of samples returned from C-type nearEarth asteroid (162173) 1999 JU3. Geochem. J. 48(6), 571-587 (2014). doi:10.2343/geochemj.2.0350

J. Takita, H. Senshu, S. Tanaka, Feasibility and accuracy of thermophysical estimation of asteroid 162173 Ryugu (1999 JU3) from the Hayabusa2 Thermal Infrared Imager. Space Sci. Rev. (2016, this issue)

Y. Tsuda, M. Yoshikawa, M. Abe, H. Minamino, S. Nakazawa, System design of the Hayabusa2_asteroid sample return mission to 1999JU3. Acta Astronaut. 91, 356-362 (2013)

J. Veverka, P. Thomas, A. Harch, B. Clark, J. Bell III., B. Carcich, J. Joseph, S. Murchie, N. Izenberg, C. Chapman, W. Merline, M. Malin, L. McFadden, M. Robinson, NEAR encounter with asteroid 253 Mathilde: overview. Icarus 140, 3-16 (1999)

F. Vilas, Spectral characteristics of Hayabusa2 near-Earth asteroid targets 1621731999 JU3 and 2001 QC34. Astron. J. 135, 1101-1105 (2008)

H. Yano, T. Kubota, H. Miyamoto, T. Okada, D. Scheeres, Y. Takagi, K. Yoshida, M. Abe, S. Abe, O. Barnouin-Jha, A. Fujiwara, S. Hasegawa, T. Hashimoto, M. Ishiguro, M. Kato, J. Kawaguchi, T. Mukai, J. Saito, S. Sasaki, M. Yoshikawa, Touchdown of the Hayabusa spacecraft at the Muses Sea on Itokawa. Science 312, 1350-1353 (2006) 